\title{
Tin chemical shift anisotropy in tin dioxide: on ambiguity of CSA asymmetry derived from MAS Spectra
}

\author{
Abil E. Aliev, Albert P. Bartók, Jonathan R. Yates
}

\section{Published version information}

Citation: AE Aliev, AP Bartók and JR Yates. "Tin chemical shift anisotropy in tin dioxide: on ambiguity of CSA asymmetry derived from MAS spectra." Solid State Nuclear Magnetic Resonance, vol. 89 (2018): 1-10.

DOI: $10.1016 /$ i.ssnmr.2017.11.002

(C)2017. This manuscript version is made available under the CC-BY-NC-ND 4.0 Licence.

This version is made available in accordance with publisher policies. Please cite only the published version using the reference above. This is the citation assigned by the publisher at the time of issuing the AAM/APV. Please check the publisher's website for any updates. 


\title{
Tin Chemical Shift Anisotropy in Tin Dioxide: On Ambiguity of CSA Asymmetry Derived from MAS Spectra
}

\author{
Abil E. Aliev, ${ }^{a *}$ Albert P. Bartók, ${ }^{b}$ Jonathan R. Yates $^{c}$ \\ ${ }^{a}$ Department of Chemistry, University College London, 20 Gordon Street, London WC1H 0AJ, U.K. \\ ${ }^{\mathrm{b}}$ Scientific Computing Department, Rutherford Appleton Laboratory, Harwell Campus, Didcot OX11 \\ 0QX, UK
}

${ }^{\mathrm{c}}$ Department of Materials, University of Oxford, Parks Road, Oxford OX1 3PH, UK

*Author for correspondence: A.E.Aliev@ucl.ac.uk

\section{RECEIVED DATE}

TITLE RUNNING HEAD: Symmetry of ${ }^{119} \mathrm{Sn}$ CSA in $\mathrm{SnO}_{2}$

KEYWORDS. Solid-state NMR; chemical shift; chemical shift anisotropy; asymmetry; oxides; nanoparticles; structure. 


\section{ABSTRACT}

Two different axial symmetries of the ${ }^{119} \mathrm{Sn}$ chemical shift anisotropy (CSA) in tin dioxide with the asymmetry parameter $(\eta)$ of 0 and 0.27 were reported previously based on the analysis of MAS NMR spectra. By analyzing the static powder pattern, we show that the ${ }^{119} \mathrm{Sn}$ CSA is axially symmetric. A nearly axial symmetry and the principal axis system of the ${ }^{119} \mathrm{Sn}$ chemical shift tensor in $\mathrm{SnO}_{2}$ were deduced from periodic scalar-relativistic density functional theory (DFT) calculations of NMR parameters. The implications of fast small-angle motions on CSA parameters were also considered, which could potentially lead to a CSA symmetry in disagreement with a crystal symmetry. Our analysis of experimental spectra using spectral simulations and iterative fittings showed that MAS spectra recorded at relatively high frequencies do not show sufficiently distinct features in order to distinguish CSAs with $\eta \approx 0$ and $\eta \approx 0.4$. The example of $\mathrm{SnO}_{2}$ shows that both the MAS lineshape and spinning sideband analyses may overestimate the $\eta$ value by as much as $\sim 0.3$ and $\sim 0.4$, respectively. The results confirm that a static powder pattern must be analysed in order to improve the accuracy of the CSA asymmetry measurements. The measurements on $\mathrm{SnO}_{2}$ nanoparticles showed that the asymmetry parameter of the ${ }^{119} \mathrm{Sn}$ CSA increases for nm-sized particles with a larger surface area compared to $\mu \mathrm{m}$-sized particles. The increase of the $\eta$ value for tin atoms near the surface in $\mathrm{SnO}_{2}$ was also confirmed by DFT calculations. 


\section{INTRODUCTION}

Compared to its solution-state counterpart, the solid-state NMR spectroscopy allows one to easily measure the chemical shift anisotropy (CSA) of spin $1 / 2$ nuclei. ${ }^{[1]}$ As with quadrupolar interactions, ${ }^{[2]}$ the anisotropy of chemical shifts can potentially provide unique information about the motional geometry at the atomistic level in various materials. ${ }^{[3]}$ The added advantage is that, unlike quadrupolar nuclei, the NMR spectra of spin $1 / 2$ nuclei are relatively easy to measure, including CSA measurements. The CSA $(\Delta \delta, \mathrm{ppm})$ and its asymmetry parameter $(\eta$, see Experimental and Computational section for definitions of $\Delta \delta$ and $\eta$ ) can also be used as a source of information about the size of nanoparticles, as at very small particle sizes the asymmetry of the environment of the surface nuclei becomes important thus affecting the measured CSA parameters due to the increase in the number of nuclei on the surface relative to the number of nuclei in the bulk. ${ }^{[4]}$ Previously, Tunstall et al. ${ }^{[4]}$ have investigated the effects of powder granule size in the range between $4-32 \mathrm{~nm}$ on parameters such as ${ }^{119} \mathrm{Sn}$ NMR CSA, linewidth and spinlattice relaxation in tin dioxide powders obtained from different thermal treatments.

Over the last 30 years, the most widely used technique applied for CSA determinations has relied on the analysis of solid-state NMR spectra acquired using magic angle spinning (MAS). General expressions were derived by Herzfeld and Berger for the intensities of sidebands observed in the MAS spectra of isolated spin half nuclei, ${ }^{[5]}$ which are now used by various program packages. ${ }^{[6-8]}$ One of the main advantages of MAS spectra compared to static lineshapes is that MAS usually allows one to resolve spinning sideband patterns for a range of species present, thus allowing one to determine CSA parameters selectively for all or nearly all species present. ${ }^{[9,10]}$ Unlike static powder spectra, the spectral overlap of the broad lines can also be avoided in MAS spectra. An additional option to vary the MAS frequency or analyse spectra measured at several different MAS frequencies are particularly useful when the analysis of several species or multiple sites present in the same spectrum is required.

The reliability of CSA measurements from MAS spectra has attracted much attention. ${ }^{[6,9-12]}$ In particular, the application of the method in the presence of strong dipolar interactions has been 
considered. ${ }^{[13]}$ There is a similarity between dipolar interactions and CSA, considering that although their effect is removed from the lineshape of the isotropic peak, both anisotropic interactions affect the intensities of the spinning sidebands. For example, homonuclear dipolar interactions between nearest ${ }^{31} \mathrm{P}$ nuclei at a distance of $4.20 \AA$ in ammonium dihydrogen phosphate have been shown to cause a deviation from axial symmetry in ${ }^{31} \mathrm{P}$ MAS spectra, while the crystal symmetry suggests an axially symmetric ${ }^{31} \mathrm{P}$ CSA. ${ }^{[13 d]}$

Frequently, distinguishing axially symmetric and asymmetric CSAs has proved problematic when using MAS spectra. ${ }^{[9]}$ As shown by Eichele, ${ }^{[6]}$ if the MAS frequency is much larger than the smallest frequency difference between two principal components, then the Herzfeld-Berger analysis may not be able to distinguish between them. According to Hodgkinson and Emsley, ${ }^{[12]}$ for the values of the asymmetry parameter less than 0.2 , static spectra provide a more reliable determination of the asymmetry parameter than MAS spectra, while the reliability of the determination of the anisotropy is always greater in spinning experiments than in static experiments. These conclusions were reached by Hodgkinson and Emsley ${ }^{[12]}$ based on computational analysis alone, without experimental verifications.

Here, we consider ${ }^{119} \mathrm{Sn}$ CSA parameters of tin(IV) oxide, $\mathrm{SnO}_{2}$, which were determined twice previously. The first CSA measurements of $\mathrm{SnO}_{2}$ were reported in 1989 by Clayden et al. ${ }^{[14]}$ The reported chemical shift anisotropy, $\Delta \delta$, was $-125 \mathrm{ppm}$ and the asymmetry parameter, $\eta$, was 0 , i.e. the ${ }^{119} \mathrm{Sn}$ CSA was shown to be axially symmetric. In their 1992 report Cossement et al., ${ }^{[15]}$ re-determined these parameters for $\mathrm{SnO}_{2}$ as $-124.5 \mathrm{ppm}$ and 0.27 , thus showing that the chemical shift tensor is not axially symmetric. No discussion of the disagreement with the results of Clayden et al. ${ }^{[14]}$ was provided and subsequent reports mostly accepted CSA parameters reported by Cossemont et al., ${ }^{[15]}$ including those using the experimental CSA parameters as a benchmark for verification of the performance of computational techniques. ${ }^{[16]}$ Note that the value of $\Delta \delta$, reported by Cossement et al. ${ }^{[15]}$ as -136 ppm for $\mathrm{SnO}_{2}$, is defined as $\Delta \delta=\delta_{33}-\delta_{11},{ }^{[13]}$ which is different from Haeberlen's definition ${ }^{[17]}$ of $\Delta \delta=\delta_{33}-\left(\delta_{11}\right.$ 
$+\delta_{22}$ ) / 2, while the same definition of $\eta$ is used by Cossement et al. ${ }^{[15]}$ and Haeberlen ${ }^{[17]}$ (see Experimental part).

Tin dioxide has a wide range of applications. Due to its superior properties in terms of electronic conductivity and thermal stability, interest in various nanoparticles based on tin dioxide has considerably increased in recent years. ${ }^{[18]}$ For example, the search of topics of publications for "tin" and "oxide" in the Web of Science database revealed >3,100 publications in 2016 and >2,100 publications between January 2017 - September 2017. Tin dioxide nanoparticles are used in catalysis, energy-saving coatings and antistatic coatings, ${ }^{[18 c, e]}$ as well as in lithium-ion batteries ${ }^{[18 b]}$ and as anti-reflection coatings in solar cells, ${ }^{[18 d]}$ in the making of gas sensors, ${ }^{[18 a]}$ optoelectronic devices, resistors and liquid crystal displays. Magnetic properties of tin oxide nanoparticles are used in magnetic resonance imaging and in data storage. It is therefore important to address the existing ambiguity in the CSA parameters of such a primary material as $\mathrm{SnO}_{2}$, so that accurate changes of the ${ }^{119} \mathrm{Sn} \mathrm{CSA}$ as a function of a nanoparticle size and environment can be monitored using solid-state NMR techniques. We note that there is only a limited number of techniques available for the characterisation of metal oxides at an atomistic level and the solid-state NMR is one the most widely used techniques in this regard. Therefore, resolving disagreement in the scientific literature is of particular importance in this case in order to remove any ambiguity that could hamper the application of solid-state NMR techniques for the characterisation of tin dioxide based materials. There is a particular interest in a size-controlled synthesis of $\mathrm{SnO}_{2}$ nanoparticles. Potentially, ${ }^{119} \mathrm{Sn}$ CSAs together with the measured signal linewidths can be used for particle size evaluations and can serve as complementary measurement to already existing techniques, such as powder X-ray powder diffraction and high-resolution transmission electron microscopy, widely used for the characterization of metal oxides. With the decrease in the particle size, the observed linewidth is expected to increase, ${ }^{[4]}$ while the CSA is expected to become more asymmetric. Severe linebroadening observed for nanoparticles of a few nanometres in size complicates the choice of the initial model for the analysis of MAS spectra compared to standard tin dioxide with normal $\sim 10 \mu \mathrm{m}$-sized particles, ${ }^{[4]}$ which shows relatively sharp lines with the 
linewidth of $\sim 150 \mathrm{~Hz}$. We have therefore undertaken the detailed analysis of tin dioxide in order to provide a correct reference point for nanoparticle studies and to identify experiments that would allow accurate determination of CSA parameters.

\section{RESULTS AND DISCUSSION}

In order to unambiguously determine the symmetry of the ${ }^{119} \mathrm{Sn}$ CSA, we have acquired a static powder pattern for $\mathrm{SnO}_{2}$. Figure 1 shows the ${ }^{119} \mathrm{Sn}$ NMR spectrum of a static $\mathrm{SnO}_{2}$ sample without any rotation.
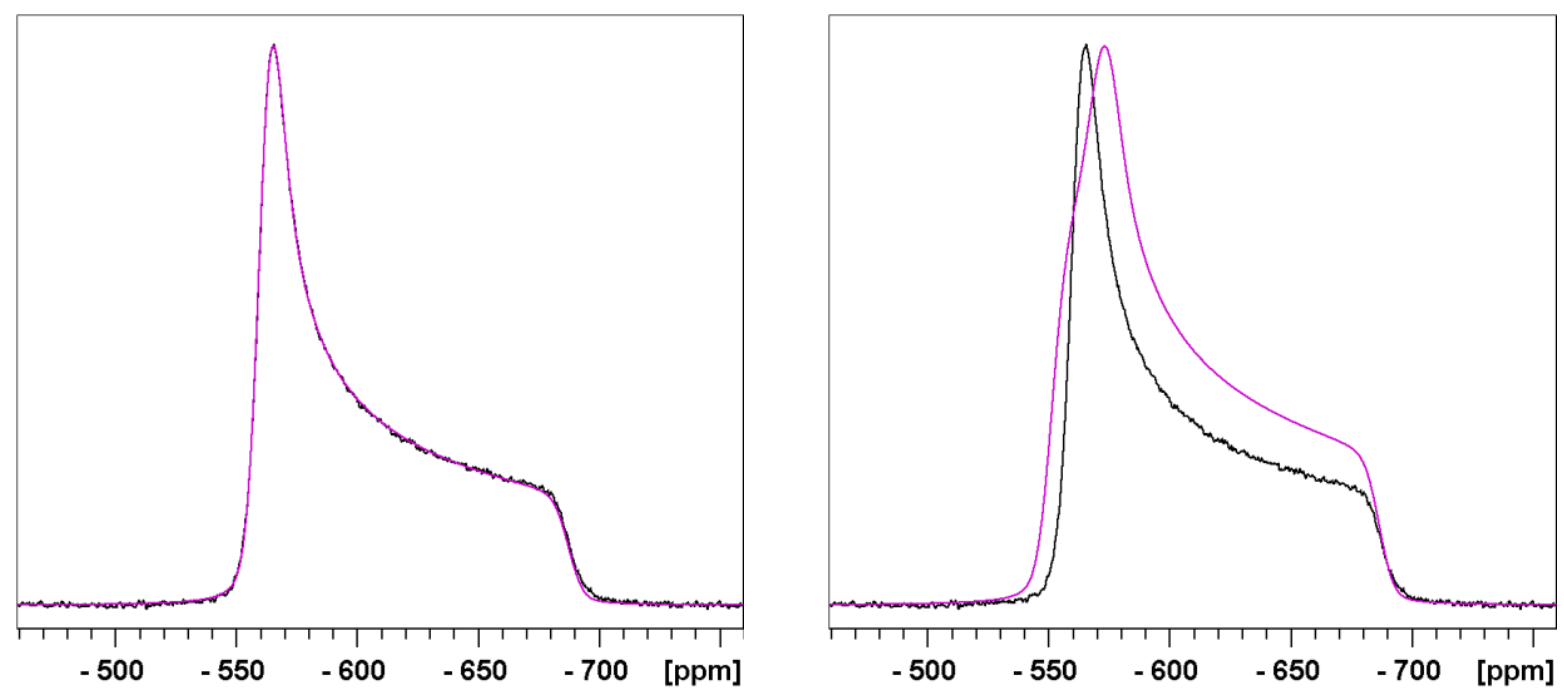

Figure 1. The experimental ${ }^{119} \mathrm{Sn} \mathrm{SSNMR}$ spectrum of $\mathrm{SnO}_{2}$ recorded without sample spinning (shown in black), overlaid with (a) the calculated spectrum with the iteratively fitted values of $\Delta \delta=-125.3 \mathrm{ppm}$ and $\eta=0$ (shown in purple) and (b) the calculated spectrum using the CSA parameters of Cossement $e t$ al. $.{ }^{[15]} \Delta \delta=-124.5 \mathrm{ppm}$ and $\eta=0.27$ (shown in purple).

Iterative fittings (Figure 1a) of the observed experimental lineshape were undertaken, in which the following error function, known as the best overlap value was maximised:

$$
\begin{aligned}
& A_{\text {dif }}=\sum_{k=1}^{N} \mid I_{k}(\exp )-I_{k}(\text { calc }) \mid \\
& A_{\text {exp }}=\sum_{k=1}^{N}\left|I_{k}(\exp )\right|
\end{aligned}
$$




$$
s_{o v}=100\left(1-\frac{A_{\text {dif }}}{A_{\text {exp }}}\right)
$$

where $A_{\text {dif }}$ is the area between the experimental and the calculated lineshapes, $A_{\exp }$ is the area of the experimental spectrum and $N$ is the number of points in the region of the experimental spectrum selected for fittings. In some of the fittings of the static powder pattern using our in-house program, the following function, referred to as the best-difference, was minimized:

$$
s_{\text {dif }}=100\left(\frac{A_{d i f}}{A_{\text {exp }}}\right)=100-s_{o v}
$$

Table 1. The values of ${ }^{119} \mathrm{Sn}$ CSA parameters of $\mathrm{SnO}_{2}$ determined from the analysis of static and MAS

\begin{tabular}{|c|c|c|c|c|c|c|}
\hline Parameter & $\begin{array}{r}\text { Static } \\
\text { (in-house) }\end{array}$ & $\begin{array}{r}\text { Static } \\
\left(\text { SoLA) }{ }^{a}\right.\end{array}$ & $\begin{array}{r}2 \mathrm{kHz} \\
\text { (SoLA) }\end{array}$ & $\begin{array}{r}3 \mathrm{kHz} \\
\text { (SoLA) }\end{array}$ & $\begin{array}{r}4 \mathrm{kHz} \\
\text { (SoLA) }\end{array}$ & $\begin{array}{r}5 \mathrm{kHz} \\
(\mathrm{SoLA})\end{array}$ \\
\hline$\delta_{\text {iso }}, \mathrm{ppm}$ & -603.81 & -603.81 & -603.81 & -603.75 & -603.83 & -603.79 \\
\hline$\Delta \delta, \mathrm{ppm}$ & -125.29 & -125.52 & -125.62 & -125.64 & -125.87 & -127.70 \\
\hline$\eta$ & 0.0 & 0.0 & 0.0 & 0.0 & 0.0 & 0.0 \\
\hline$\delta_{11}, \mathrm{ppm}$ & -562.03 & -561.94 & -561.94 & -561.87 & -561.88 & -561.22 \\
\hline$\delta_{22}, \mathrm{ppm}$ & -562.06 & -561.99 & -561.94 & -561.87 & -561.88 & -561.22 \\
\hline$\delta_{33}, \mathrm{ppm}$ & -687.34 & -687.48 & -687.55 & -687.51 & -687.74 & -688.91 \\
\hline$\Omega, \mathrm{ppm}$ & 125.31 & 125.54 & 125.62 & 125.64 & 125.86 & 127.69 \\
\hline$\kappa$ & 0.99951 & 1.00 & 1.0 & 1.0 & 1.0 & 1.00 \\
\hline overlap, \% & 98.57 & 99.10 & 94.86 & 95.66 & 95.26 & 95.40 \\
\hline
\end{tabular}
(frequency shown in $\mathrm{kHz}$ ) lineshapes. The programs used (see Experimental) are indicated in brackets.

\footnotetext{
${ }^{a}$ Bruker curve fitting program SoLA (Solid Lineshape Analysis, version 2.2.4)
}

The region between -438.7 and $-768.7 \mathrm{ppm}$ was selected for fittings with $N=6,052$. The best-fit values of the CSA parameters were $\Delta \delta=-125.3 \pm 0.9 \mathrm{ppm}$ and $\eta=0.0 \pm 0.011$ (Table 1). Uncertainties were estimated via fittings of 10 differently processed spectra, including the variation of the exponential line broadening factor between $0-50 \mathrm{~Hz}$, using two different programs (see also Figure S1 in Supporting 
Information showing the dependence of the error function on the value of $\eta$ ). For comparison, we also show calculated lineshapes with $\Delta \delta=-124.5 \mathrm{ppm}$ and $\eta=0.27$ determined by Cossemont et al. ${ }^{[15]}$ (Figure 1b), which clearly does not reproduce the experimentally observed lineshape.

Density functional calculations were carried out with CASTEP within the GIPAW (Gauge Including Projector Augmented Waves) formalism to predict CSA parameters (Tables 2 and 3). For the two experimentally determined structures, ${ }^{[19]}$ computed values of CSA show little variance with different exchange-correlation functionals. Thus, these functionals appear to be sufficiently similar in predicting NMR parameters for a fixed geometry. For the optimised structures, however, there is a slightly larger difference which can be attributed to the different equilibrium geometries. Notably, the environment of the $\mathrm{Sn}$ atoms in the LDA-relaxed structure (Table 2) deviates more from the fully symmetrical arrangement due to the somewhat larger difference in the two types of Sn-O bond lengths (Table 2), but lattice vibrations even at room temperature are expected to be larger than this difference. For example, the anisotropic displacement parameters in $\mathrm{SnO}_{2}$ are known to reach $4.4 \times 10^{-3} \AA^{2}$ and $6.6 \times 10^{-3} \AA^{2}$ for Sn and $\mathrm{O}$ atoms, respectively. ${ }^{[19 \mathrm{~b}]}$ As for the optimised lattice constants (Table 2), the observed functionaldependent changes are in agreement with the expectation that the most commonly used GGA (generalised gradient approximation) functional proposed by Perdew, Burke, and Ernzherhof (PBE) almost always overestimates the lattice constants of solids, while LDA (local density approximation) consistently underestimates them. ${ }^{[20]}$ In both cases, the typical errors amount to $1 \%-2 \%$ of the lattice parameters. ${ }^{[20]}$ The PBEsol ${ }^{[20 \mathrm{a}]}$ functional is the revised version of PBE specifically adapted for solids, which is known to yield lattice constants in better agreement with experiments than the original PBE functional. ${ }^{[20 \mathrm{c}]}$ Indeed, our results in Table 2 confirm that lattice constants predicted by PBEsol are in better agreement with the experimental data than those from PBE or PW91. There appears to be a good correlation for the calculated asymmetry parameter in four optimised structures (Table 3) with either the area $a b$ or volume $a b c$, i.e. with the increase of either the transverse area $a b$ or the lattice volume $a b c$, the calculated asymmetry parameter decreases. 
Table 2. Lattice parameters and nearest-neighbour Sn-O bond distances in Ångstroms in experimental and DFT-relaxed $\mathrm{SnO}_{2}$ structures.

\begin{tabular}{rcccc}
\hline Structure & $a$ & $c$ & Sn-O & Sn-O 2 \\
\hline$(\mathrm{I})^{[19 \mathrm{a}]}$ & 4.7380 & 3.1865 & 2.052 & 2.058 \\
$(\mathrm{II})^{[19 \mathrm{~b}]}$ & 4.7374 & 3.1864 & 2.058 & 2.048 \\
LDA & 4.7158 & 3.1881 & 2.058 & 2.041 \\
PBE & 4.8187 & 3.2335 & 2.086 & 2.090 \\
PW91 & 4.8136 & 3.2321 & 2.085 & 2.087 \\
PBEsol & 4.7592 & 3.2083 & 2.067 & 2.061 \\
\hline
\end{tabular}

Table 3. ${ }^{119} \mathrm{Sn}$ CSA parameters in $\mathrm{SnO}_{2}$ predicted by DFT calculations, using various exchange correlation functionals and structural parameters.

\begin{tabular}{ccccc}
\hline \multirow{2}{*}{ Structure } & \multicolumn{2}{c}{ Chemical shift anisotropy, $\Delta \delta($ in ppm) / Asymmetry parameter, $\eta$} \\
& LDA & PBE & PW91 & PBEsol \\
\hline$(\mathrm{I})^{[19 \mathrm{a}]}$ & $-141.3 / 0.09$ & $-139.8 / 0.09$ & $-138.9 / 0.09$ & $-142.3 / 0.08$ \\
$(\mathrm{II})^{[19 \mathrm{~b}]}$ & $-127.2 / 0.09$ & $-125.8 / 0.09$ & $-125.2 / 0.09$ & $-128.0 / 0.10$ \\
optimised & $-124.8 / 0.18$ & $-129.4 / 0.04$ & $-130.2 / 0.04$ & $-129.2 / 0.09$ \\
\hline
\end{tabular}

Solid-state NMR spectra were also recorded and analyzed at different MAS frequencies (Figure 2). As starting values of CSA parameters in iterative fittings, we used $\Delta \delta=-125.3 \mathrm{ppm}$ and $\eta=0$, as derived from the analysis of the static powder pattern. The best-fit values are summarized in Table 1 . These showed good agreement with the values measured from the static spectrum with only a small difference in the $\Delta \delta$ value, which increases on increasing the MAS frequency. 

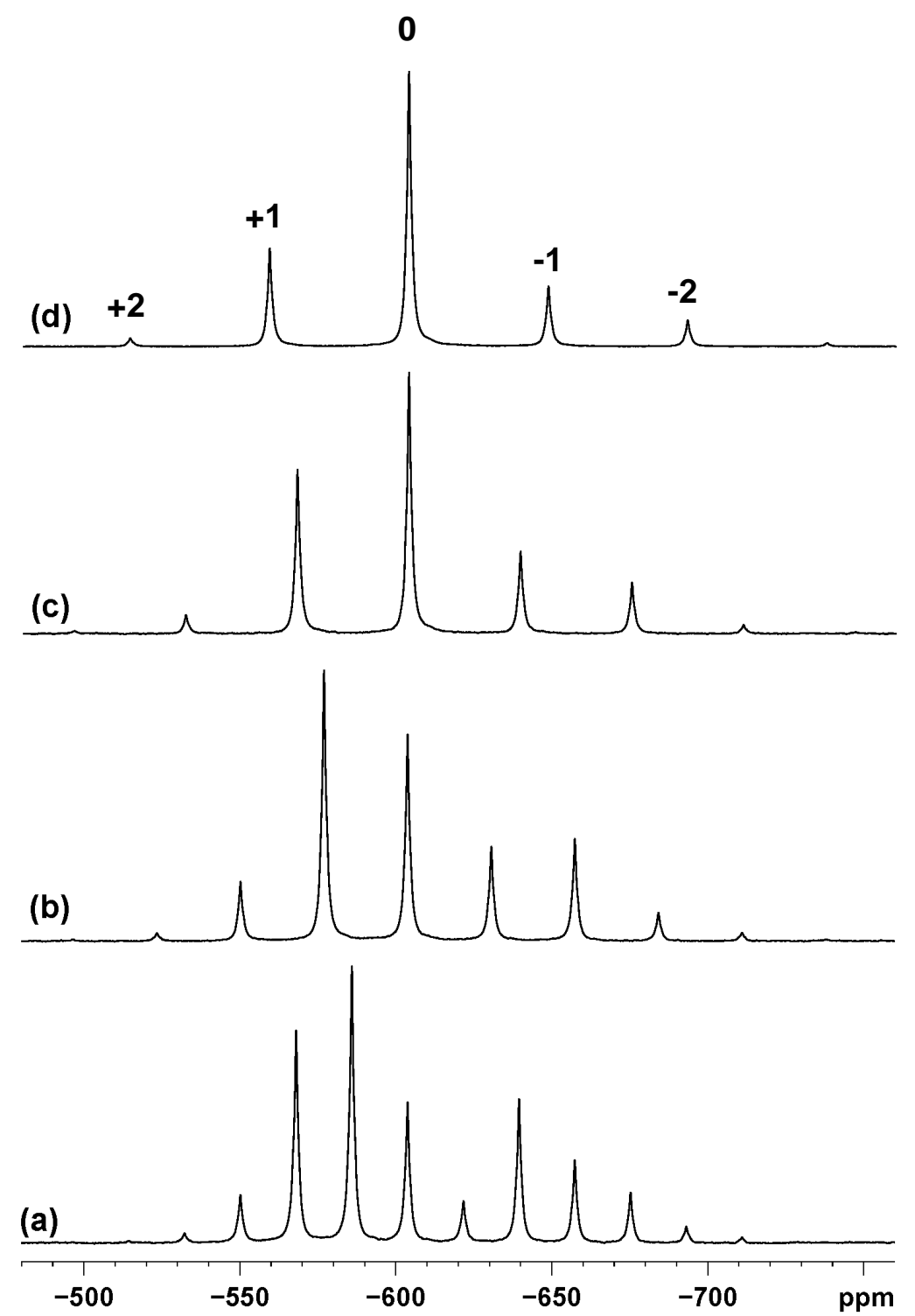

Figure 2. The experimental ${ }^{119} \mathrm{Sn} \mathrm{SSNMR}$ spectra of $\mathrm{SnO}_{2}$ recorded at MAS frequencies of (a) $2 \mathrm{kHz}$, (b) $3 \mathrm{kHz}$, (c) $4 \mathrm{kHz}$ and (d) $5 \mathrm{kHz}$. The spinning sideband labelling ( \pm 1 and \pm 2 ) is shown in (d) relative to the isotropic peak labelled as 0 .

Cossement et al. ${ }^{[15]}$ used the MAS frequency of $5.1 \mathrm{kHz}$ (at $9.4 \mathrm{~T}$ corresponding to a $400 \mathrm{MHz}{ }^{1} \mathrm{H}$ frequency) for CSA measurements. They started their analysis with the initial estimate of the CSA span $\left(\delta_{33}-\delta_{11}\right)$ as $-200 \mathrm{ppm}$ and the asymmetry parameter $\eta$ as 0.5 . We undertook additional fittings of the spectrum recorded at the MAS frequency of $5 \mathrm{kHz}$ at $7.05 \mathrm{~T}$ (Figure $2 \mathrm{~d}$ ) with the starting value of $\Delta \delta$ as $-126 \mathrm{ppm}$ and $\eta$ as 0.5 . The best-fit values found from iterative fittings were $\Delta \delta=-126.29 \mathrm{ppm}$ and $\eta=$ 0.285. The agreement between the experimental and the calculated lineshapes estimated as the best 
overlap value, $s_{\text {ov }}$ (see equations (1)-(3)), was $95.56 \%$, which is better than $95.40 \%$ for $\Delta \delta=-127.70$ ppm and $\eta=0$ (Table 1). On fitting the spectrum recorded at the MAS frequency of $2 \mathrm{kHz}$ at $7.05 \mathrm{~T}$ (Figure 2a) with the starting value of $\Delta \delta$ as $-126 \mathrm{ppm}$ and $\eta$ as 0.5 , the best-fit values were $\Delta \delta=-125.24 \mathrm{ppm}$ and $\eta=0.179$, with the $s_{\text {ov }}$ value of $95.22 \%$, i.e. better than $94.86 \%$ for $\Delta \delta=-125.62 \mathrm{ppm}$ and $\eta=0$ (Table 1). These were somewhat surprising results, as the best-fit spectra were established using an "incorrect" set of parameters. We therefore compared fitted lineshapes. At the MAS frequency of $5 \mathrm{kHz}$ spectra with $\eta=0.0$ and $\eta=0.285$ were very similar to each other (Figures S2 and S3 in Supporting Information). This is in contrast with static powder patterns shown in Figure 1. Even at the MAS frequency of $2 \mathrm{kHz}$, spectra showed only small changes in relative intensities of spinning sidebands for $\eta$ values of 0.0 and 0.179 (Figures S2 and S3 in Supporting Information). This result suggests that there is a more general problem with the use of MAS spectra for CSA measurements, i.e. the MAS spectra of tin dioxide at 2 and $5 \mathrm{kHz}$ do not show sufficient distinct features for distinguishing the solution with $\eta=0$ from that with $\eta \approx 0.2$ or $\eta \approx 0.3$.

To follow the sensitivity of the MAS spectrum to the asymmetry parameter, we have simulated ${ }^{119} \mathrm{Sn}$ MAS spectra at $5 \mathrm{kHz}$, where the reduced ${ }^{119} \mathrm{Sn}$ chemical shift anisotropy $\left(\delta_{\text {red }}=\delta_{33}-\delta_{\text {iso }}=-84 \mathrm{ppm}\right)$ is kept constant, while $\eta$ is changed between 0 and 1 (Figure 3). These simulations show that there is hardly any noticeable change in spinning sideband intensities for $\eta$ values between 0 and 0.3 . On going from $\eta$ $=0.3$ to $\eta=0.4$, only a small increase in intensity is observed for the -1 sideband at approximately -650 ppm. The intensity of this sideband continues to increase gradually on increasing the $\eta$ value further. These results indicate to an insensitivity of the MAS experiment to $\eta$ values less than 0.3 , and only little sensitivity at $\eta$ values 0.3 and 0.4 

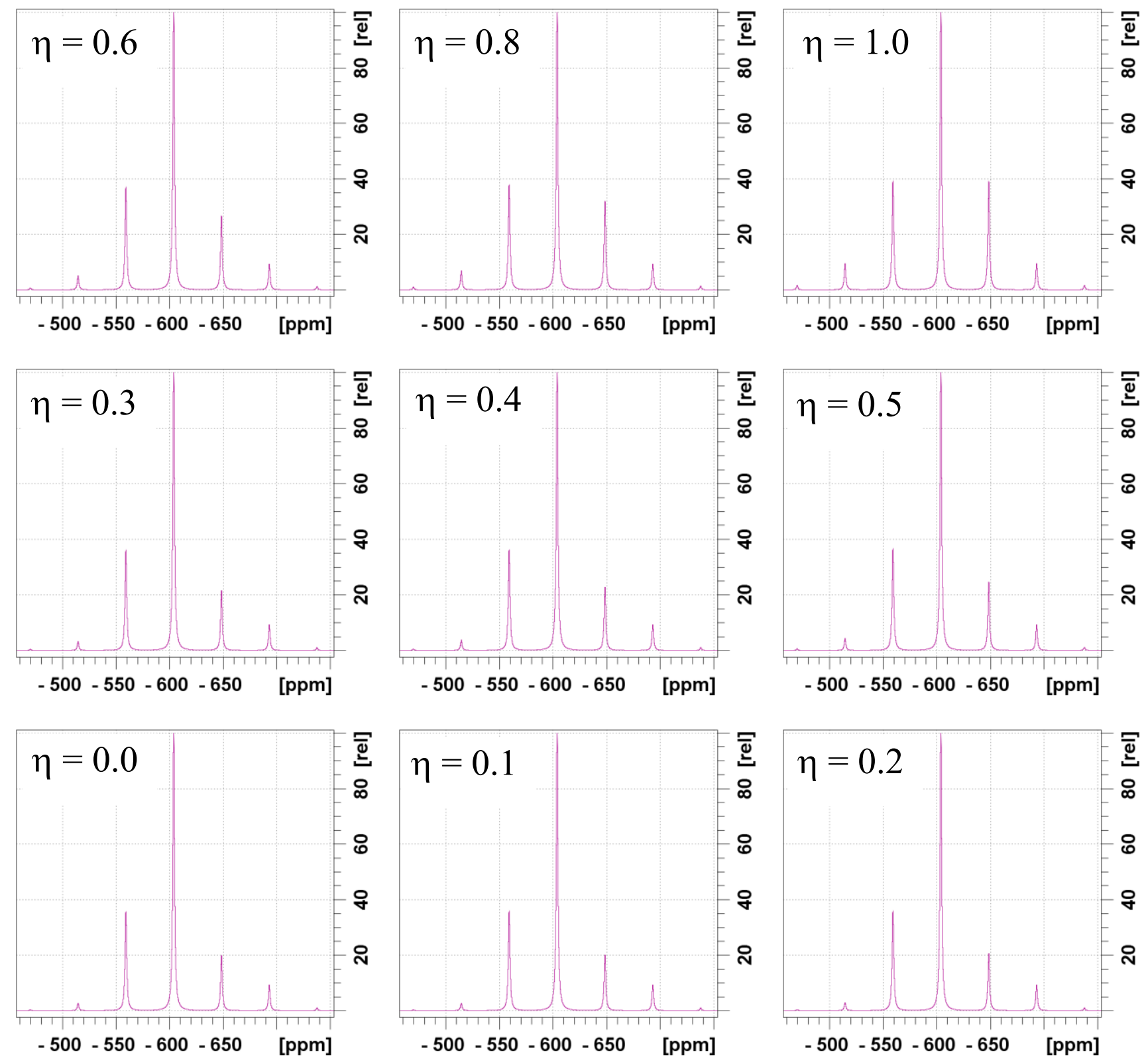

Figure 3. The spectral simulations of MAS spectra at $5 \mathrm{kHz}$, where the reduced ${ }^{119} \mathrm{Sn}$ chemical shift anisotropy $\left(\delta_{\text {red }}=\delta_{33}-\delta_{\text {iso }}=-84 \mathrm{ppm}\right)$ is kept constant, while $\eta$ is changed between 0 and 1 .

As the error function used in our above lineshape fittings of MAS spectra fails to favour the correct solution with $\eta=0$ at relatively low MAS frequency of $2 \mathrm{kHz}$, we have considered a more traditional Herzfeld-Berger method, which relies on the relative intensities of spinning sidebands. ${ }^{[5]}$ One of the most mature programs, HBA, was used for this purpose. ${ }^{[6]}$ HBA uses a Marquardt-Levenberg routine for iterative minimisation of the error function, $s_{\text {fit }}$, defined as: 


$$
S_{f i t}=\left\{\sum_{k=1}^{N} \frac{\left[I_{k}(\text { calc })-I_{k}(\exp )\right]^{2}}{(N-p)}\right\}^{1 / 2}
$$

where $I$ denotes the intensity of spinning sidebands and the isotropic peak, $N$ is the total number of experimental peaks (including spinning sidebands and the isotropic peak), intensities of which are selected for the analysis and $p$ is the number of optimized parameters. A statistical analysis of errors is carried out using dimensionless Herzfeld-Berger parameters $\mu$ and $\kappa::^{[5]}$

$$
\begin{aligned}
& \mu=\left(v_{\mathrm{L}} / v_{\mathrm{R}}\right)\left(\delta_{11}-\delta_{33}\right) 10^{-6} \\
& \kappa=3\left(\delta_{22}-\delta_{\text {iso }}\right) /\left(\delta_{11}-\delta_{33}\right)
\end{aligned}
$$

where $v_{L}$ and $v_{R}$ are Larmor and MAS frequencies, respectively. This analysis involves the calculation of a matrix $\mathbf{B}$, the elements of which are calculated using the following equation:

$$
B_{i j}=\sum\left[\frac{\partial I_{k}(\text { calc })}{\partial p_{i}}\right]\left[\frac{\partial I_{k}(\text { calc })}{\partial p_{j}}\right]
$$

where $p_{i, j}$ are the parameters $\mu$ and $\kappa, \partial I_{k}($ calc $)$ is the change in calculated intensity when parameters $\mu$ and $\kappa$ are changed slightly from their optimized best-fit values by \pm 0.2 and \pm 0.05 , respectively, and the summation involves all experimentally observed sidebands. The ratio $R_{\mathrm{i}}$ of conditional, $s_{\text {cond }}$, and marginal, $s_{\text {marg, }}$, standard deviations is calculated using following equations:

$$
\begin{aligned}
& s_{\text {cond }}\left(p_{\mathrm{i}}\right)=s_{\text {fit }} \times\left(B_{i i}\right)^{-1 / 2} \\
& s_{\text {marg }}\left(p_{\mathrm{i}}\right)=s_{\text {fit }} \times\left[\left(B^{-1}\right)_{i i}\right]^{1 / 2} \\
& R_{\mathrm{i}}=s^{2}{ }_{\text {marg }}\left(p_{\mathrm{i}}\right) / s_{\text {cond }}^{2}\left(p_{\mathrm{i}}\right)
\end{aligned}
$$

As shown previously, for a parameter $p_{\mathrm{i}}$ to be reasonably well defined by the experimental data, the ratio $R_{\mathrm{i}}$ must be close to $1 .^{[9]}$ Thus, while the error function $s_{\text {fit }}$ reflects how well the predicted spinning sideband intensities match the corresponding experimental intensities, the values of additional parameters from 
equations (9)-(11) allow us to evaluate how well the optimized parameters are defined by the experimental data for a given model. ${ }^{[9]}$

As with the lineshape fittings of MAS spectra, the analysis of the integral intensities of experimental spinning sidebands (Table S1 in Supporting Information) favours the asymmetric CSA model with two different $\eta$ values of 0.27 and 0.41 at MAS frequencies of 2 and $5 \mathrm{kHz}$, respectively, based on the value of the error function, $s_{\mathrm{fit}}$, alone. The improvements compared to the axially symmetric CSA model is significant at both MAS frequencies and the value of $s_{\text {fit }}$ was reduced nearly by a factor of 2 . At both 2 and $5 \mathrm{kHz}$ MAS frequencies, however, the calculated ratios $R(\kappa)$ indicate that the $\eta=0$ value is better defined by experimental data than either $\eta=0.27$ or $\eta=0.41$ (Table S1 in Supporting Information).

We have also considered a possibility of fitting intensities of spinning sidebands from multiple MAS spectra in a single iterative analysis. The joint analysis of all 4 spectra recorded at different MAS frequencies (Figure 2) in this manner led to $\eta=0.24(\Delta \delta=-125.3 \mathrm{ppm})$. For MAS spectra analysed separately at 2, 3, 4 and $5 \mathrm{kHz}$, the best-fit $\eta$ values were $0.27,0.16,0.21$ and 0.41 , respectively. Therefore, in none of the cases the correct value of $\eta=0$ was deduced as the best-fit value from either separate or joint analysis of spinning sideband intensities in MAS spectra. Furthermore, the cross-examination via independent fittings of four different MAS spectra shows significant differences in the best-fit $\eta$ values varying between $0.16-0.41$. This indicates to the presence of systematic errors in CSA measurements from MAS spectra. Note that throughout their study of the reliability of the determination of the anisotropy and the asymmetry parameter of the chemical shift interaction using the Cramér-Rao lower bounds, Hodgkinson and Emsley ${ }^{[12]}$ assumed that systematic errors are negligible in comparison with the random error. The example of tin dioxide considered above shows that deriving the CSA asymmetry from fittings of MAS spectra will be systematically incorrect as long as the MAS frequency is smaller than the differences between the principal components $\left(\delta_{11}-\delta_{22}\right.$, according to the definition of $\left.\eta\right) .{ }^{[6]}$ Then, in the case of the axial symmetry, when the differences between the principal components is zero, the MAS 
frequency must be infinitely small in order to ensure that the source of systematic errors is removed, thus suggesting the use of static lineshape for the analysis. The requirement for the MAS frequency to be smaller than the difference $\left(\delta_{11}-\delta_{22}\right)$ could arise from the requirement to sample the frequency region between $\delta_{11}$ and $\delta_{22}$ as densely as possible in order to minimize systematic errors.

The spectral simulations presented in Figure 3 show that MAS spectra recorded at relatively high frequencies do not show sufficiently distinct features in order to distinguish CSAs with $\eta \approx 0$ and $\eta \approx 0.4$. In addition, the example of $\mathrm{SnO}_{2}$ considered above confirms that the spinning sideband analysis of MAS spectra for CSA measurements may overestimate the $\eta$ value by as much as $\sim 0.4$, if the choice of the best solution is based on the error function alone. The latter reflects how well the fitted data matches the experiment, but does not account for how well the fitted parameters are defined by the experiment. The use of a single MAS spectrum recorded at a relatively high MAS frequency for the determination of CSA parameters therefore presents a wider concern in terms of misidentifying the CSA symmetry, if the reported $\eta$ values are in the range $0 \leq \eta \leq 0.4$. Further revision may be needed in each case using the analysis of static powder patterns.

Solid-state 2D NMR techniques are also widely applied for measurements of CSA parameters. ${ }^{[10,21]}$ Despite differences in various 2D techniques developed, they usually share the common goal of displaying an MAS-like spectrum along one dimension and a static or slow-spinning-sideband powder pattern along the other dimension. ${ }^{[10,21]}$ In the light of the above discussion, these $2 \mathrm{D}$ techniques may prove useful for extracting static or slow-spinning-sideband powder patterns when multiple inequivalent sites or a range of different species are present. However, it is also well recognized that the applications of 2D techniques for CSA measurements are restricted, especially when multiple sites present in the system have the same or similar isotropic chemical shifts or when a very large number different sites are present in the solid material under study.

Despite the lack of the high-resolution, the static powder patterns can be used to distinguish axially symmetric $(\eta=0)$ and nearly axially symmetric $(\eta \approx 0.1)$ CSAs in favourable cases with a narrow intrinsic 
linewidth. A representative example in this regard is the static ${ }^{31} \mathrm{P}$ NMR spectrum of the monoclinic polymorph of triphenyl phospine oxide (TPPO, Figures S4 and S5 in Supporting information), where the inspection of the experimental static lineshape alone is sufficient to identify the axial asymmetry of the ${ }^{31} \mathrm{P}$ CSA (see also Figure S6, showing the static ${ }^{119} \mathrm{Sn}$ NMR spectrum of $\mathrm{SnO}_{2}$ recorded at $16.44 \mathrm{~T}$ ). In addition, the results of the CSA analysis collected in Table S2 show that the ratio $R(\kappa)$ of conditional and marginal standard deviations [equation (11), ${ }^{[9]}$ for interconversions of $\kappa$ and $\eta$, see equation (19)] does not favour the correct solution with $\eta \approx 0.1$ over $\eta=0$ on analysing ${ }^{31}$ P MAS spectra. For example, at the MAS frequency of $2 \mathrm{kHz}, R(\kappa)=16$ for $\eta=0.094$ and $R(\kappa)=1$ for $\eta=0$, i.e. the $R(\kappa)$ value suggests that the axial symmetry of the CSA with $\eta=0$ is better defined by the experimental MAS spectrum than the correct solution with $\eta=0.094$, known from the analysis of the static powder pattern. It appears that the $R$ parameter may systematically favour the $\eta=0$ case over that with small but nonzero value of $\eta$ when an MAS spectrum is used for determining the CSA parameters. This additional example further emphasizes the importance of static lineshape measurements for establishing the correct symmetry of the chemical shift anisotropy.

We now consider the symmetry of the ${ }^{119} \mathrm{Sn}$ CSA in tin(IV) oxide in relation to its solid-state structure. In metal oxides, chemical shift parameters of metal atoms reflect the local symmetry of the oxygen polyhedral environments, which in turn is determined by the crystal structure. The less symmetric arrangement of oxygens surrounding a metal atom in a unit cell is expected to lead to a large chemicalshift anisotropy. For example, the ${ }^{119} \mathrm{Sn} \mathrm{CSA}$ is $-125 \mathrm{ppm}$ in $\mathrm{SnO}_{2}$, while it is $-975 \mathrm{ppm}$ in $\mathrm{SnO}$ (for comparison of surroundings of tin atoms in $\mathrm{SnO}_{2}$ and $\mathrm{SnO}$ unit cells, see Figure 1 in Cossemont et al. ${ }^{[15]}$ ), i.e. the more spherical coordination of the tin atom in $\mathrm{SnO}_{2}$ leads to a smaller CSA compared to that in SnO.

Three-dimensionally, a chemical shift anisotropy can be schematically presented using an ellipsoid (Figure 4a), the shape of which is determined by semi-principal axes $\boldsymbol{a}, \boldsymbol{b}$ and $\boldsymbol{c}$, which also represent distances from the centre of the ellipsoid to its surface along the $x, y$, and $z$ axes, respectively. In this case, 
$\boldsymbol{a}=\delta_{11}, \boldsymbol{b}=\delta_{22}$ and $\boldsymbol{c}=\delta_{33}$. In the case of axial asymmetry, all three distances from the origin of the ellipsoid to its surface along axes $\boldsymbol{a}, \boldsymbol{b}$ and $\boldsymbol{c}$ are different, while in the case of axial symmetry we expect two of the distances to be equal, but different from the third distance: $\boldsymbol{a}=\boldsymbol{b} \neq \boldsymbol{c}$. Such a presentation allows us to relate the symmetry of the CSA to the symmetry of the environment surrounding the tin atom, as observed in crystallographic studies.

For the solid-state structure of tin(IV) oxide, ${ }^{[19]}$ the space group is $\mathrm{P} 4{ }_{2} / m n m .{ }^{[19 \mathrm{~b}]}$ The cell lengths are $a$ $=b=4.7374 \AA$ and $c=3.1864 \AA$. The cell angles are $\alpha=\beta=\gamma=90^{\circ}$. As shown in Figure $4 \mathrm{~b}$, the $\mathrm{Sn}-\mathrm{O}$ distances in the plane parallel to the $a b$ plane of the unit cell are equal in length $(2.048 \AA)$. In the diagonal plane of the unit call with the central tin atom and four oxygen atoms, all four Sn-O distances are equal $(2.058 \AA)$. The three different $\mathrm{O}-\mathrm{Sn}-\mathrm{O}$ bond angles are $78.52^{\circ}, 101.48^{\circ}$ and $90.00^{\circ}$. Note that Clayden et al. ${ }^{[14]}$ have attributed the observed anisotropy of the ${ }^{119} \mathrm{Sn}$ CSA in tin dioxide to the differences in the bond angles.

The principal axis system (PAS) of the ${ }^{119} \mathrm{Sn}$ chemical shift tensor was deduced from CASTEP calculations in this work (Figure $4 b$ ). Due to the mirror planes, the PAS of the ${ }^{119} \mathrm{Sn}$ chemical shift tensor is expected to be orientated along [ $\left[\begin{array}{lll}1 & 1 & 0\end{array}\right],\left[\begin{array}{lll}1 & 1 & 0\end{array}\right]$ and $\left[\begin{array}{lll}0 & 0 & 1\end{array}\right]$. Thus, the CASTEP calculation correctly predicts the orientations of the $x, y$ and $z$ axes of the PAS as parallel to [ $\left.\begin{array}{llll}1 & 1 & 0\end{array}\right],\left[\begin{array}{lll}1 & 1 & 0\end{array}\right]$ and $\left[\begin{array}{lll}0 & 0 & 1\end{array}\right]$ directions, respectively. Although the crystal symmetry determines the PAS in this case, it does not allow us to estimate the relative values of the three principal components, i.e. whether $\delta_{11}=\delta_{22} \neq \delta_{33}$ or $\delta_{11} \neq$ $\delta_{22} \neq \delta_{33}$. However, as the $y$ axis of the PAS is along the Sn-O bond and the $x$ axis of the PAS is along the direction joining two Sn atoms $6.7 \AA$ apart, there is no reason to expect that the electronic environment is the same in these directions and therefore the $\delta_{11}$ and $\delta_{22}$ components are equal. This is in agreement with the CASTEP predictions of $\eta$, as it is found in the range of $0.04-0.18$ (depending on the DFT method used) for the optimised structures and in the range $0.08-0.10$ for the structures from diffraction measurements (Table 2). Nevertheless, as the experimental value is 0 , there is a good possibility that fast (compared to the NMR timescale) small-angle motions are present at room temperature which average 
the measured value of $\eta$ to 0 . Such a possibility does indeed exist, provided that the tip of $z$ axis of the PAS (Figure 4b) undergoes a full rotation, e.g. a wobbling type of motion. ${ }^{[2 \mathrm{c}, 22]}$ This is very similar to the well-studied examples from ${ }^{2} \mathrm{H}$ NMR, where ${ }^{2} \mathrm{H}$ quadrupole coupling constant and its asymmetry show dependence on the geometry and the amplitude of the fast small-angle motions. ${ }^{[2 c]}$
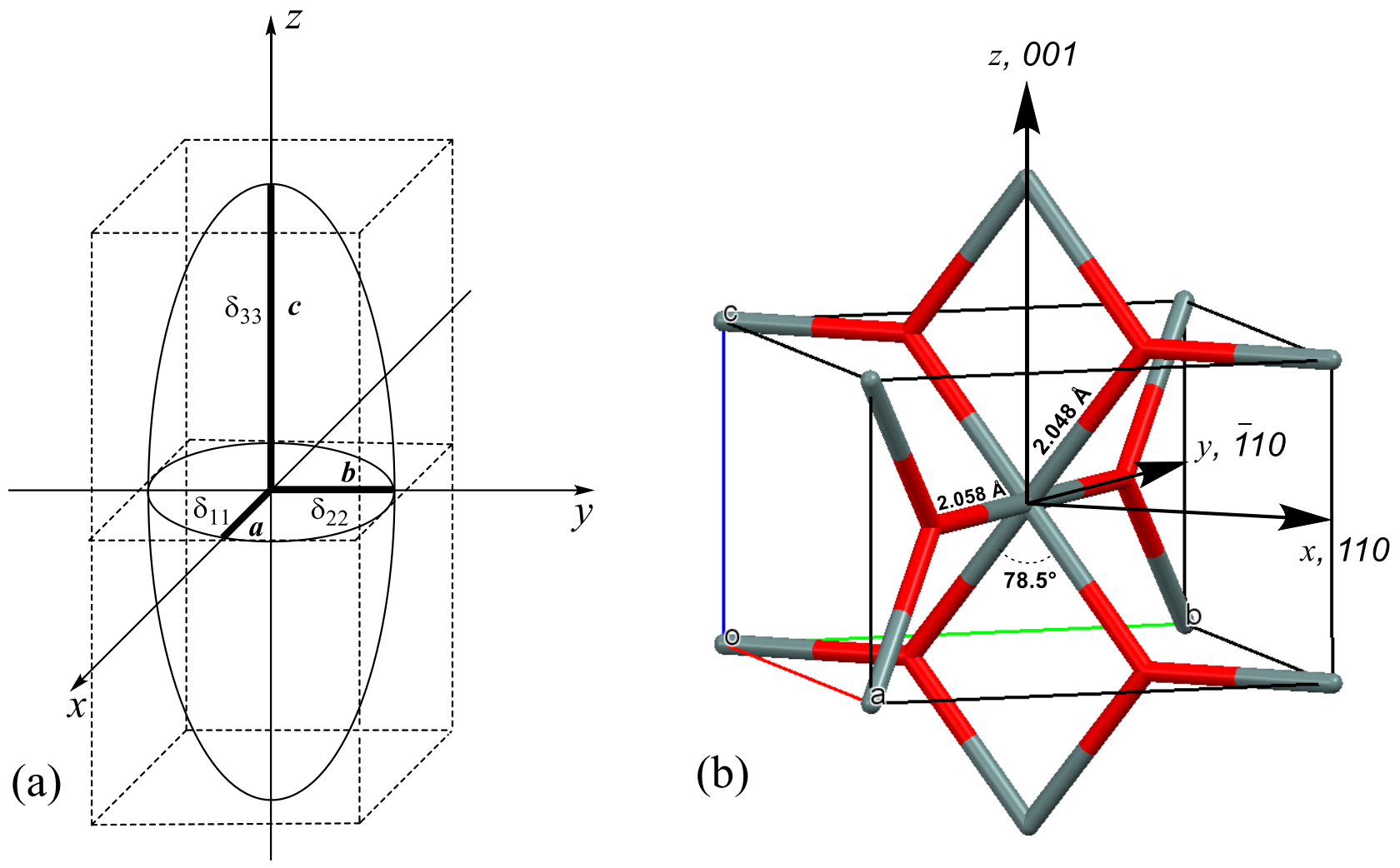

Figure 4. (a) A schematic presentation of CSA as an ellipsoid, the shape of which is determined by distances $\boldsymbol{a}=\delta_{11}, \boldsymbol{b}=\delta_{22}$ and $\boldsymbol{c}=\delta_{33}$ from the centre of the ellipsoid to its surface along the $x, y$, and $z$ axes; (b) A unit cell of $\mathrm{SnO}_{2}$, as determined from neutron diffraction powder data. ${ }^{[19 \mathrm{~b}]}$ An orientation of the ${ }^{119} \mathrm{Sn}$ CSA PAS as predicted by CASTEP calculations is shown, where the $x, y$ and $z$ axes of the PAS are parallel to [ $\left[\begin{array}{lll}1 & 1 & 0\end{array}\right],\left[\begin{array}{lll}\overline{1} & 1 & 0\end{array}\right]$ and [ $\left[\begin{array}{lll}0 & 0 & 1\end{array}\right]$ directions, respectively.

An example illustrating the effect of small-angle fast motions on the asymmetry of the CSAs is shown in Figure 5. In the fast motional regime in the NMR timescale, the NMR spectrum can be analysed in the same manner as that in the absence of the motions (see, for example, references $[3,22,23]$ ), with the difference that the CSA will be determined by the motionally averaged anisotropy, $\Delta \delta^{*}$, and the motionally averaged asymmetry parameter, $\eta^{*}$. In Figure 5, we show that depending on whether the principal $z$ axis 
of the CSA tensor undergoes a fast out-of-plane wobbling motion (simulated as a jump motion of the $z$ axis of the CSA with a $10^{\circ}$ step between 36 equally spaced, equally populated orientations on the surface of a cone with a cone half-angle of $9^{\circ}$ ) or an in-plane librational motion (around the axis perpendicular to the $z$-axis of the CSA with the angle between the orientations of the $z$-axis of the CSA at the extrema $+9^{\circ}$ and $-9^{\circ}$ ) the motionally averaged CSA will be axially symmetric and asymmetric, respectively.

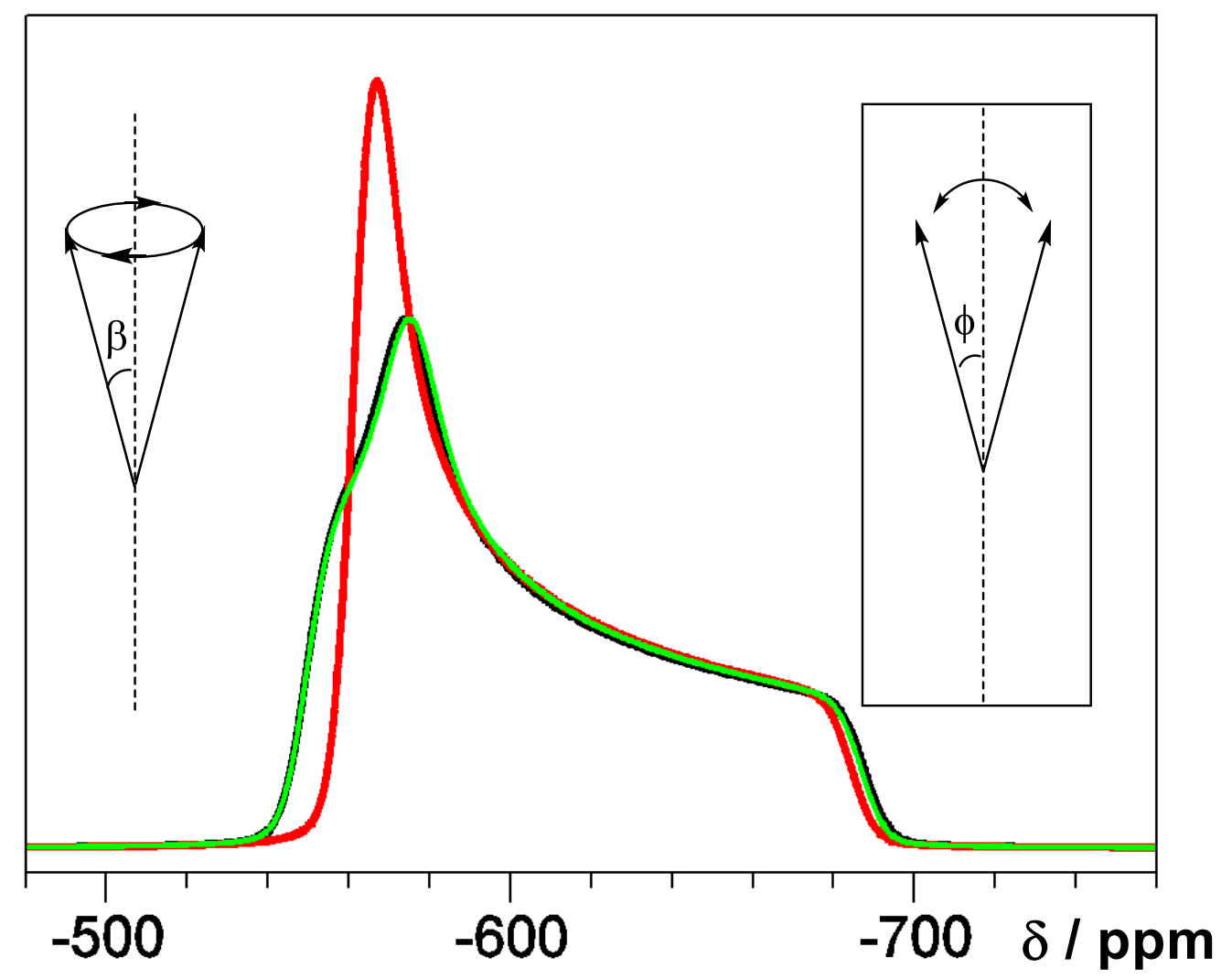

Figure 5. The spectral simulations of static CSA lineshapes in the presence of a fast out-of-plane wobbling motion (shown in red; illustrated on the left) and an in-plane librational motion (shown in green; illustrated on the right). The static CSA lineshape in the absence of any motion is shown in black. The static CSA parameters $\Delta \delta=-126 \mathrm{ppm} / \eta=0.300$ are motionally averaged to $\Delta \delta^{*}=-120.9 \mathrm{ppm} / \eta^{*}=0$ and $\Delta \delta^{*}=$ $124.6 \mathrm{ppm} / \eta^{*}=0.315$ by wobbling and librational motions, respectively, when $\beta=\phi=9^{\circ}$.

The relevance of this example in the case of tin dioxide is that the amplitude of a full-rotation ( $\beta$ in Figure 5) will affect the value of the motionally averaged anisotropy, $\Delta \delta^{*}$ i.e. the higher the amplitude, 
the smaller the value of $\left|\Delta \delta^{*}\right|$. However, the value of $\beta$ is dependent on the temperature and should be at least $\sim 6^{\circ}$ in order to lead to an experimentally significant $\sim 2 \mathrm{ppm}$ decrease in the $\left|\Delta \delta^{*}\right|$ value, i.e. very high-temperature measurements may be required in the case of rigid metal oxides. Unlike $\Delta \delta^{*}$, even at a very small, but non-zero value of $\beta$ the expected value of $\eta^{*}$ is 0 and any additional increase of $\beta$ has no further effect on $\eta^{*}$. We note that the presence of thermal vibrations in $\mathrm{SnO}_{2}$ was shown by crystallographic studies. In particular, Bolzan et al. ${ }^{[19 b]}$ analysed 11 different metal dioxides, including $\mathrm{SnO}_{2}$, which adopt the rutile-type structure. They showed that the magnitude of the thermal vibrations of the atoms are correlated with the size of the cell which is relatively large in $\mathrm{SnO}_{2}$ compared to other oxides considered. ${ }^{[19 b]}$ They also showed that thermal vibrations are strongly anisotropic for both metal and oxygen atoms. The metal atom displacements in the $a b$ plane were larger than the displacement along the $c$ axis (see Figure 4). It is therefore possible that the small-angle motions present in tin dioxide could be responsible for the axial symmetry of the chemical shift tensor measured experimentally. As the effect of small-angle motions is of general interest, particularly in terms of the possibility that the $\eta=0$ case is often encountered due to the dynamics rather than the crystal symmetry, it would be beneficial to employ advanced techniques for the joint analysis of both the XRD and NMR results. Further detailed scrutiny of these subtle effects on the chemical shift tensor parameters, especially using the recently developed path integral molecular dynamics (PIMD) simulations, ${ }^{[24]}$ will certainly be of general interest.

As discussed above, the CSA parameters are expected to be sensitive to the particle size. In order to study the influence of the particle size on the ${ }^{119} \mathrm{Sn}$ CSA parameters, Tunstall et al. ${ }^{[4]}$ used MAS spectra recorded at about $6 \mathrm{kHz}$ at $11.75 \mathrm{~T}$. Unlike tin dioxide considered above, for nano-sized particles with a relatively high surface area, the observed NMR spectra can be considered as a sum of spectra due to a very large number of sites with different CSA parameters. Therefore, the measured values of CSA parameters will be affected by the structural disorder present in the system. To distinguish parameters measured for structurally inhomogeneous systems, we denote the CSA parameters measured for nanoparticles as $\langle\Delta \delta\rangle$ and $\langle\eta\rangle$. From the figures presented by Tunstall et al., ${ }^{[4]}$ the $\langle\Delta \delta\rangle /\langle\eta\rangle$ values 
determined were $-131 \mathrm{ppm} / 0.11,-125 \mathrm{ppm} / 0.31,-113 \mathrm{ppm} / 0.66,-124 \mathrm{ppm} / 0.49,-123 \mathrm{ppm} / 0.32$ and -126 ppm/0.0 for $\mathrm{SnO}_{2}$ particles with the average sizes of 4, 5, 8, 16, 32 and $\sim 10,000 \mathrm{~nm}$, respectively, with the uncertainty of $\pm 12 \mathrm{ppm}$ for $\langle\Delta \delta\rangle$ and \pm 0.1 for $\langle\eta\rangle$. Thus, they observed significant changes in the CSA parameters, especially in the asymmetry parameter, as a function of the size of $\mathrm{SnO}_{2}$ particles. The increase of the isotropic linewidth with the decrease of the particle size was also observed, with the measured approximate values of $650,750,1250,1700$ and $1550 \mathrm{~Hz}$ for particle sizes of 32, 16, 8, 5 and $4 \mathrm{~nm}$, respectively, with the estimated uncertainty of $\pm 10 \%$. The maximum deviation from bulk values occurring at a particle size of $8 \mathrm{~nm}$ was observed in both the ${ }^{119} \mathrm{Sn}$ CSA asymmetry parameter and in the value of the ${ }^{119} \mathrm{Sn}$ NMR spin-lattice relaxation time. From the combined analysis of solid-state NMR and Raman results, it was concluded that the $8 \mathrm{~nm}$ particles are situated at the transition from bulk-dominated to surface-dominated samples. ${ }^{[4]}$ However, it must be noted that the CSA parameters were determined from MAS spectra recorded at about $6 \mathrm{kHz}(\sim 32 \mathrm{ppm})$, whereas the difference between the reported $\left\langle\delta_{11}\right\rangle$ and $\left\langle\delta_{22}\right\rangle$ values (-567 and $-576 \mathrm{ppm}$, respectively) is only $9 \mathrm{ppm}$ for $4 \mathrm{~nm}$ particles. Additionally, the linewidth increases with the decrease of the particle size, reaching $8.3 \mathrm{ppm}$ for $4 \mathrm{~nm}$ particles, which is nearly the same as the difference between the reported $\left\langle\delta_{11}\right\rangle$ and $\left\langle\delta_{22}\right\rangle$ values. In this case, both the high MAS frequency and the broad linewidth are likely to adversely affect the accuracy of CSA asymmetry measurements from MAS spectra, since $\langle\eta\rangle$ is defined as $\left(\left\langle\delta_{22}\right\rangle-\left\langle\delta_{11}\right\rangle\right) /\left(\left\langle\delta_{33}\right\rangle-\left\langle\delta_{1 \sigma o}\right\rangle\right)$.

Tin dioxide nanoparticles with an estimated size of $\sim 10 \mathrm{~nm}$ were available to us, showing a linewidth of $630 \pm 20 \mathrm{~Hz}$ ( $150 \pm 5$ for tin dioxide considered above; both measured from the spectra recorded at the MAS frequency of $12 \mathrm{kHz}$ at $7.05 \mathrm{~T}$ ). From iterative fittings of the static lineshape (Figure 6a), the bestfit CSA parameters were $\langle\Delta \delta\rangle=-127 \pm 3 \mathrm{ppm}$ and $\langle\eta\rangle=0.2 \pm 0.1$. The MAS spectra of $\mathrm{SnO}_{2}$ nanoparticles recorded at 3, 4 (Figure $6 \mathrm{~b}$ ) and $5 \mathrm{kHz}$ were also analysed using full lineshape fittings (Table 4). The bestfit values of $\langle\eta\rangle$ were $0.6 \pm 0.1$ at $3 \mathrm{kHz}$ and $0.5 \pm 0.1$ at 4 and $5 \mathrm{kHz}$. 
a)
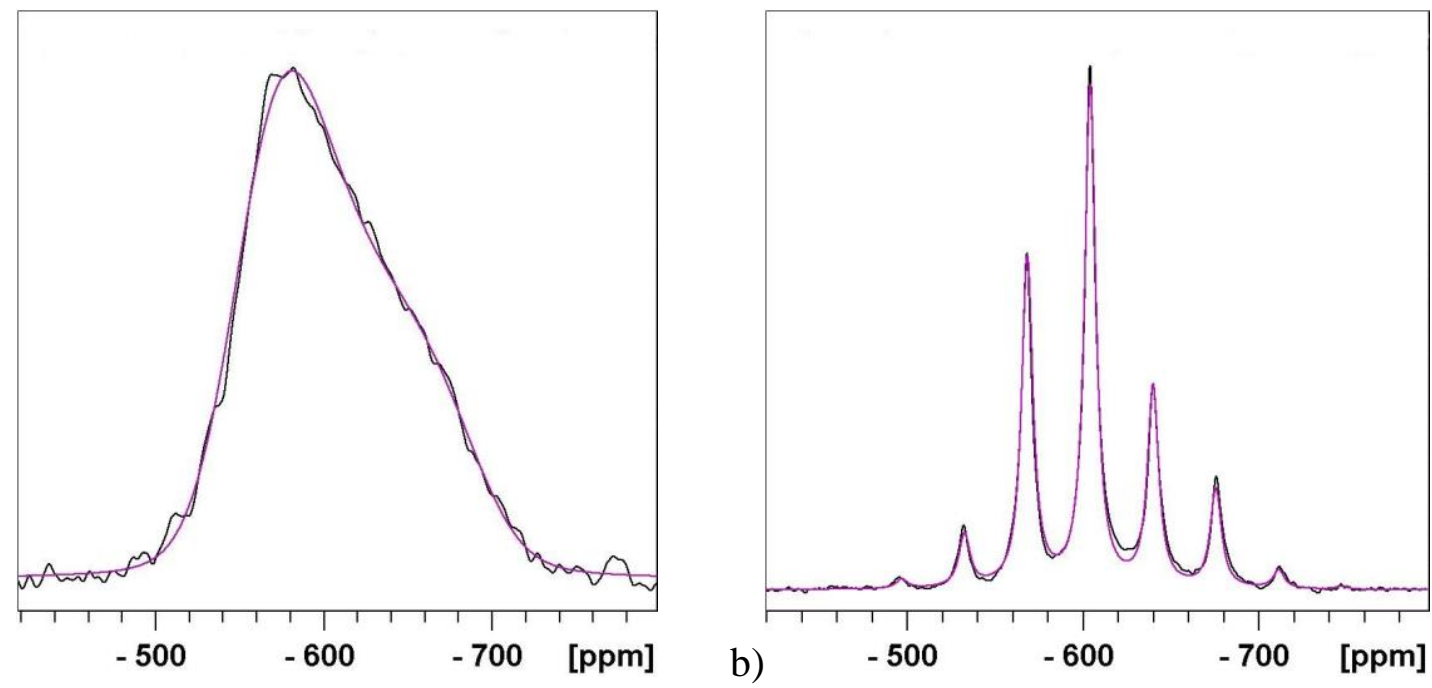

Figure 6. The experimental ${ }^{119} \mathrm{Sn}$ SSNMR spectra (shown in black) of $\mathrm{SnO}_{2}$ nanoparticles recorded (a) without sample spinning, and (b) at MAS frequency of $4 \mathrm{kHz}$. Spectra are overlaid with the calculated lineshapes (shown in purple) with the iteratively fitted values of $\langle\Delta \delta\rangle=-127 \mathrm{ppm}$ and $\langle\eta\rangle=0.20$ in (a) and $\langle\Delta \delta\rangle=-127 \mathrm{ppm}$ and $\langle\eta\rangle=0.52$ in (b).

Although the agreement between the CSA parameters determined from spectra at different MAS frequencies is satisfactory, the values of $\eta$ are nevertheless significantly higher than that derived from the static lineshape $(\langle\eta\rangle=0.2 \pm 0.1)$. This is similar to the results discussed above for tin dioxide, for which the analysis of MAS spectra leads to $\eta$ values higher than that determined from static powder patterns by 0.2 - 0.4. As apparent from the results shown in Table 4, the best-fit value of the $\delta_{33}$ component from both MAS and static spectra agree well, while the difference in $\left(\left\langle\delta_{11}\right\rangle-\left\langle\delta_{22}\right\rangle\right)$ values is significant: 18 ppm from the static spectrum and $\sim 45 \mathrm{ppm}$ from the MAS spectra. Since the asymmetry parameter is defined as $\langle\eta\rangle=\left(\left\langle\delta_{22}\right\rangle-\left\langle\delta_{11}\right\rangle\right) /\left(\left\langle\delta_{33}\right\rangle-\left\langle\delta_{\text {iso }}\right\rangle\right)$, the nearly doubled value of $\left(\left\langle\delta_{22}\right\rangle-\left\langle\delta_{11}\right\rangle\right)$ at a constant value of $\left(\left\langle\delta_{33}\right\rangle-\left\langle\delta_{\text {iso }}\right\rangle\right)$ leads to significant increase in $\eta$ values derived from MAS spectra compared to that from the static powder pattern. As the predicted difference of $\left(\left\langle\delta_{22}\right\rangle-\left\langle\delta_{11}\right\rangle\right)$ is only $\sim 2 \mathrm{kHz}$ at $7.05 \mathrm{~T}$, it is clear that MAS frequencies significantly slower than $2 \mathrm{kHz}$ are needed in order to adequately sample the spectrum for accurate measurements of CSA asymmetries. However, slow MAS experiments are limited by the linewidth of the isotropic peak, thus making static lineshapes a preferred choice for CSA measurements. 
Table 4. The values of ${ }^{119} \mathrm{Sn}$ CSA parameters of $\mathrm{SnO}_{2}$ nanoparticles determined from the analysis of static and MAS (frequency shown in $\mathrm{kHz}$ ) lineshapes. The chemical shift range between $-400 \mathrm{ppm}$ and -850 ppm was selected for fittings.

\begin{tabular}{rrrrr}
\hline Parameter & Static & $\mathbf{3 ~ k H z}$ & $\mathbf{4 ~ k H z}$ & $\mathbf{5 ~ k H z}$ \\
\hline$\left\langle\delta_{\text {iso }}\right\rangle, \mathrm{ppm}$ & $-604^{\mathrm{a}}$ & -604 & -604 & -604 \\
$\langle\Delta \delta\rangle, \mathrm{ppm}$ & -128 & -129 & -127 & -132 \\
$\langle\eta\rangle$ & 0.2 & 0.6 & 0.5 & 0.5 \\
$\left\langle\delta_{11}\right\rangle, \mathrm{ppm}$ & -553 & -535 & -540 & -539 \\
$\left\langle\delta_{22}\right\rangle, \mathrm{ppm}$ & -571 & -587 & -584 & -580 \\
$\left\langle\delta_{33}\right\rangle, \mathrm{ppm}$ & -689 & -691 & -689 & -691 \\
$\langle\Omega\rangle, \mathrm{ppm}$ & -571 & 156 & 149 & 153 \\
$\langle\kappa\rangle$ & 0.7 & 0.3 & 0.4 & 0.4 \\
\hline Best overlap, \% & 96.47 & 93.70 & 95.56 & 96.01 \\
\hline
\end{tabular}

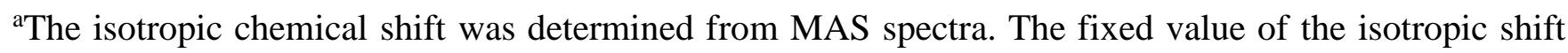
was used in fittings of the static powder pattern.

Theoretical studies of the effect of particle size on NMR parameters were also undertaken by computing the CSA parameters of $\mathrm{Sn}$ atoms in various low-index, relaxed stoichiometric surfaces of $\mathrm{SnO}_{2}$. $\mathrm{Slabs}$ were generated using the PBE equilibrium lattice parameters from the bulk structure, obtaining the $\left[\begin{array}{lll}1 & 0 & 0\end{array}\right]$, $\left[\begin{array}{lll}0 & 0 & 1\end{array}\right],\left[\begin{array}{lll}1 & 0 & 1\end{array}\right]$ and [ $\left[\begin{array}{lll}1 & 1 & 0\end{array}\right]$ surfaces. These are the surfaces previously shown to be low-energy, ${ }^{[25}$ and therefore are expected to provide a good representation of the nanocluster surfaces. To minimise interactions between the surfaces, the thickness of each slab was chosen to ensure that bulk-like NMR parameters are reproduced for the atoms in the centre regions, and $6 \AA$ vacuum was used to separate the surfaces. As illustrated in Figure 7, there is a significant change in both the CSA and asymmetry for atoms near the surface. It is clear that the measured parameters depend on the exact ratio of the different atomic 
environments making up the cluster, which we do not attempt to quantify here, but we can conclude that the increase in the asymmetry parameter is indeed confirmed.
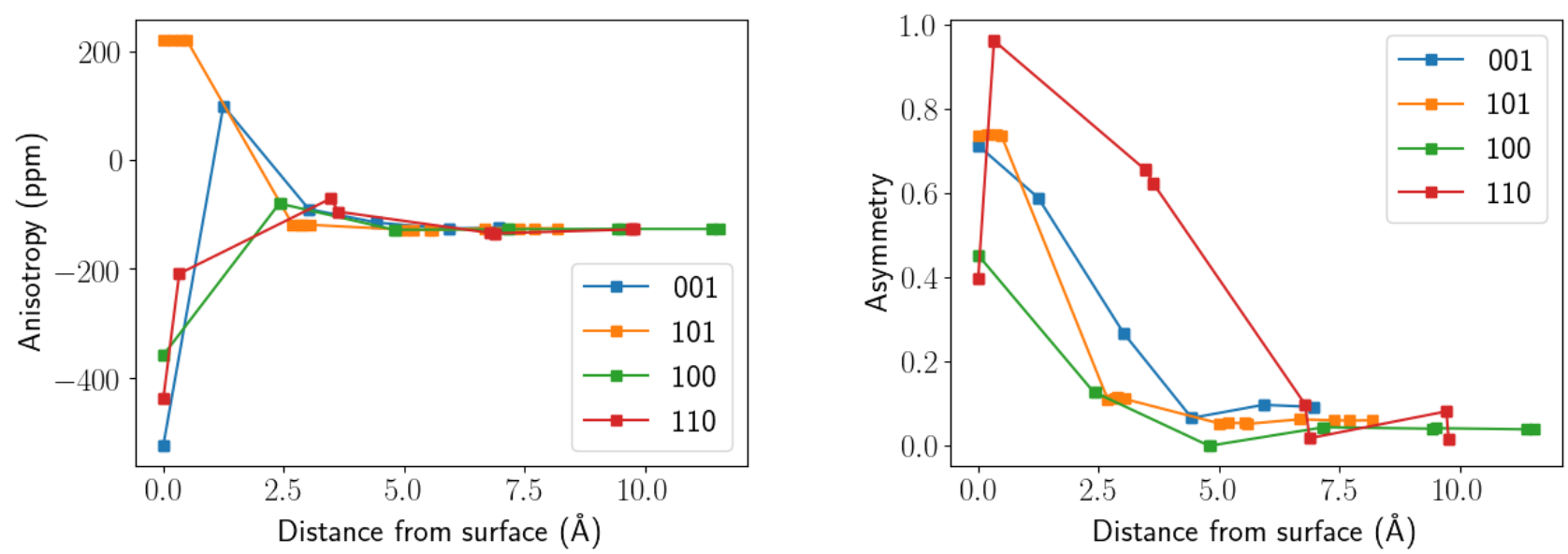

Figure 7. The DFT-predicted CSA (left panel) and asymmetry (right panel) of ${ }^{119} \mathrm{Sn}$ atoms as a distance from the surface. The lines provide a guide for the eye. Note that the large positive anisotropy value $(229 \mathrm{ppm})$ of the surface Sn atoms on the [ $\left.\begin{array}{lll}1 & 0 & 1\end{array}\right]$ surface correspond to a high asymmetry value $(0.80)$, therefore the sign of the anisotropy is less meaningful, as it can easily change with a relatively small perturbation.

\section{CONCLUDING REMARKS}

Two different sets of ${ }^{119}$ Sn CSA parameters with $\eta=0$ and $\eta=0.27$ were reported previously for tin(IV) oxide based on the analysis of MAS NMR spectra. ${ }^{[14,15]}$ By analyzing the static powder pattern, we show that the asymmetry parameter is 0 . Periodic scalar-relativistic density functional calculations of NMR parameters suggest a nearly axial symmetry of the ${ }^{119} \mathrm{Sn} \mathrm{CSA}$ in $\mathrm{SnO}_{2}$. The principal axis system of the ${ }^{119} \mathrm{Sn}$ chemical shift tensor was also deduced from these calculations. Our analysis of MAS spectra using spectral simulations and iterative fittings showed that MAS spectra recorded at relatively high frequencies do not show sufficiently distinct features in order to distinguish CSAs with asymmetry parameters $\eta \approx 0$ and $\eta \approx 0.4$. The analysis of MAS spectra recorded at $2 \mathrm{kHz}$ or $5 \mathrm{kHz}$ using iterative fittings of either the full lineshape or the spinning sideband intensities also failed to lead to the correct solution, known from 
the analysis of the static powder pattern. We have shown that a static powder pattern must be analysed in order to improve the accuracy of the CSA asymmetry measurements when $\eta$ falls within a range between $0 \leq \eta<0.4$. As judged by only very small changes in simulated MAS spectra, the relative insensitivity of MAS spectra to $\eta$ values in the range $0 \leq \eta<0.4$ is likely to be of general nature. Therefore, the use of MAS spectra for CSA determinations with the asymmetry parameter in the range between 0 and 0.4 presents a wider concern in terms of misidentifying the CSA symmetry, especially when a single spectrum recorded at a relatively high MAS frequency is considered. Potentially, a significant proportion of the reported results in the literature may be inaccurate. The implications of the latter could be particularly detrimental for verification of computational techniques. A representative example in this regard is the work by Alkan et al., ${ }^{[16]}$ where the incorrect set of experimental CSA parameters for tin dioxide ${ }^{[15]}$ was used as a benchmark for the calculated values by different DFT methods.

We note that distinguishing the axially symmetric CSA from that which is nearly axially symmetric can be critically important for both structural and dynamics studies. As discussed above using the example of small-angle motions (Figure 5), accurate knowledge of the CSA symmetry allows one to deduce such subtle dynamics in the solid state as the geometry and the amplitude of motion not available from other techniques. The case of the measured axially symmetric CSA is also of practical methodological importance as fast (compared to the NMR timescale) small-angle dynamics may lead to motional averaging of the CSA asymmetry parameter to 0 , in disagreement with the known crystal symmetry often measured at very low temperatures.

An example of structural importance is from nanoparticles. While for micrometre-sized particles the contribution from the surface area can be ignored, in the case of nanometre-sized particles the importance of the environment of the surface nuclei is becoming important. Thus, changes in the ${ }^{119} \mathrm{Sn}$ CSA parameters are expected on going from micrometre-sized particles to nanoparticles. Note that the measured ${ }^{119} \mathrm{Sn}$ CSA parameters are statistically averaged in nanoparticles due to the presence of different bulk and surface tin sites. In addition, as illustrated computationally (Figure 7) in this work, there are also 
differences in tin sites depending on the distance from the surface. In principle, two-dimensional experiments could be useful when a handful of different sites are present in the system with comparable populations of the sites. In nanoparticles, however, the number of different sites approach nearly infinity. The feasible solution is therefore to measure parameters for nanoparticles in the same manner as for micrometre-sized tin dioxide and then look at their deviation from the CSA values measured for bulk tin dioxide. As apparent from the shape of the isotropic peak shown in Figure $6 \mathrm{~b}$ for $\mathrm{SnO}_{2}$ nanoparticles (see also spectra reported by Tunstall et al. ${ }^{[4]}$, MAS does not allow to resolve multiple tin sites in varying environments. However, the linewidth of the isotropic peak in MAS spectra can serve as a good indication of how wide the distribution of structural inhomogeneity is in a given sample of $\mathrm{SnO}_{2}$ nanoparticles and this was explored successfully by Tunstall et al. ${ }^{[4]}$ As pointed above, a size-controlled synthesis of $\mathrm{SnO}_{2}$ nanoparticles is of primary practical importance and the CSA measurements could serve as a useful tool in this regard. Our initial results obtained from the analysis of the static powder pattern suggest that the asymmetry parameter of the ${ }^{119} \mathrm{Sn}$ chemical shift tensor increases for nm-sized $\mathrm{SnO}_{2}$ nanoparticles compared to $\mu \mathrm{m}$-sized $\mathrm{SnO}_{2}$. This is in agreement with the previously reported results of Tunstall et al. ${ }^{[4]}$ and the results of DFT calculations presented in this work.

\section{EXPERIMENTAL AND COMPUTATIONAL SECTION}

Nuclear Magnetic Resonance Spectroscopy. Tin(IV) oxide of 99\% purity was purchased from Fisher

Scientific and was used without further treatment. Solid-state ${ }^{119}$ Sn NMR spectra were measured at 111.92 MHz on a Bruker Avance 300 spectrometer with 7.05 T wide-bore magnet at ambient probe temperature using a standard Bruker $4 \mathrm{~mm}$ double-resonance MAS probe. Tin(IV) oxide was packed into a zirconia rotor of $4 \mathrm{~mm}$ external diameter and spun at the MAS frequencies of 2-12 kHz with stability better than $\pm 3 \mathrm{~Hz}$. The same sample and probe were used for recording a ${ }^{119} \mathrm{Sn}$ NMR spectrum without any rotation. Solid-state ${ }^{119} \mathrm{Sn}$ NMR spectra with and without high-power proton decoupling were recorded using the following acquisition conditions: ${ }^{119} \mathrm{Sn} 90^{\circ}$ pulse duration $=2.3 \mu \mathrm{s}$; recycle delay $=250 \mathrm{~s}$; number of 
transients 128 - 640. For "static" measurements without sample rotation, Hahn echo (with an echo delay of $15 \mu \mathrm{s}$ ) and single-pulse sequences were employed. As expected for a powder pattern with a relatively narrow width of $\sim 18 \mathrm{kHz}$, no significant changes were observed between spectra recorded with singlepulse and Hahn echo sequences. The spectrum recorded with the Hahn echo sequence was used in our iterative fittings. The ${ }^{119} \mathrm{Sn}$ chemical shifts are given relative to tetramethyltin $\mathrm{SnMe}_{4}$, which were calibrated using tetrakis(trimethylstannyl)methane $\mathrm{C}\left(\mathrm{SnMe}_{3}\right)_{4}(48.2 \mathrm{ppm}) .{ }^{[26]}$

The static powder spectrum of tin dioxide was analysed using our in-house Fortran program, which is a modified version of the FASTPOWDER program, originally written for ${ }^{2} \mathrm{H}$ lineshape simulations. ${ }^{[27]}$ Simplex and simulated annealing algorithms ${ }^{[28]}$ were used for optimisation of spectral parameters, as described previously for ${ }^{2} \mathrm{H}$ lineshapes. ${ }^{[29]}$ The best difference value $s_{\text {dif }}$ (see equation (4)) was minimised in the iterative search for best-fit values. Static and MAS spectral lineshape fittings were also carried out using the Bruker curve fitting program SoLA (Solid Lineshape Analysis, version 2.2.4), which is part of their standard NMR software package TopSpin (version 3.2). As an estimate of the degree of agreement between the calculated and experimental lineshapes, the best overlap value $\left(s_{\text {ov }}\right.$, in $\left.\%\right)$ is used in SoLA (see equation (3) above), which is maximized in iterative fittings. The simplex algorithm is used in lineshape fittings. Unlike quasi-global optimization techniques, ${ }^{[28]}$ the simplex algorithm (as well as the Levenberg-Marquardt algorithm used in the HBA program $)^{[6]}$ are known to lead to local minima in the error function depending on the starting values used. All the calculated static (by SoLA and modified FASTPOWDER) and MAS lineshapes (by SoLA) were convoluted by Lorentzian and Gaussian linebroadening functions with the linewidth parameters of $L B$ and $G B$, respectively. Typical best-fit values of $L B / G B$ were $167 / 1135 \mathrm{~Hz}$ in fittings of the static lineshape of $\mathrm{SnO}_{2}, 180 / 100 \mathrm{~Hz}$ in fittings of MAS spectra of $\mathrm{SnO}_{2}, 1187 / 5958 \mathrm{~Hz}$ in fittings of the static lineshape of $\mathrm{SnO}_{2}$ nanoparticles, $1580 / 0 \mathrm{~Hz}$ in fittings of MAS spectra of $\mathrm{SnO}_{2}$ nanoparticles.

The above mentioned modified version of the FASTPOWDER program ${ }^{[27,29]}$ was also used for spectral simulations of static CSA lineshapes in the presence of fast small-angle motions. 
In the case of the ${ }^{119} \mathrm{Sn}$ chemical shift anisotropy considered in this work with $\left(\delta_{33}-\delta_{\text {iso }}\right)<0$, parameters are defined as follows:

$$
\begin{aligned}
& \text { Principal CSA components } \delta_{11} \geq \delta_{22} \geq \delta_{33} \\
& \text { Isotropic value } \delta_{\text {iso }}=\left(\delta_{11}+\delta_{22}+\delta_{33}\right) / 3 \\
& \text { Reduced chemical shift anisotropy } \Delta \delta_{\text {red }}=\delta_{33}-\delta_{\text {iso }} \\
& \text { Chemical shift anisotropy } \Delta \delta=\delta_{33}-\left(\delta_{11}+\delta_{22}\right) / 2=3 \Delta \delta_{\text {red }} / 2 \\
& \text { Asymmetry parameter } \eta=\left(\delta_{22}-\delta_{11}\right) /\left(\delta_{33}-\delta_{\text {iso }}\right) \text { with } 0 \leq \eta \leq 1 \text {; } \\
& \text { Span } \Omega=\delta_{11}-\delta_{33} \\
& \text { Skew } \kappa=3\left(\delta_{22}-\delta_{\text {iso }}\right) / \Omega \text { with }(-1 \leq \kappa \leq 1)
\end{aligned}
$$

Here, the principal components of the chemical shift tensor, $\delta_{11}, \delta_{22}$ and $\delta_{33}$, are defined such that the highest frequency (the least shielded) component is labelled as $\delta_{11}$, while $\delta_{33}$ corresponds to the lowest frequency (the most shielded) component. Unless otherwise specified, we use the definition of the chemical shift anisotropy according to equation (15), which is different from that of the reduced chemical shift anisotropy (see equation (14)) used in some solid-state NMR programs (e.g., SIMPSON, ${ }^{[11]}$ SPINEVOLUTION ${ }^{[30]}$ and Bruker curve fitting program SoLA). The two parameters reflecting the asymmetry of the chemical shift tensor, $\kappa$ and $\eta$, can be interconverted using the following relationships:

$$
\eta=\left[\delta_{\text {iso }}-\delta_{11}+(\kappa \Omega / 3)\right] /\left(\delta_{33}-\delta_{\text {iso }}\right)
$$

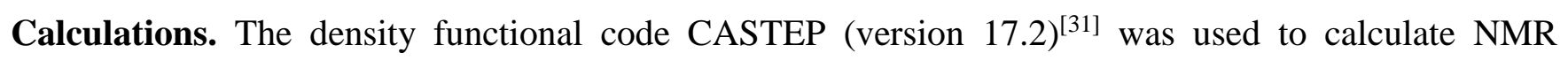
parameters with a selection of local density and generalized gradient approximation exchange-correlation functionals. ${ }^{[32]}$ Dispersion corrections were not included in the calculations. A plane-wave basis set with $870 \mathrm{eV}$ cutoff energy was used in conjunction with on-the-fly generated ultrasoft pseudopotentials, including scalar relativistic effects within the zero-order regular approximation (ZORA). ${ }^{[33]}$ The Brillouin zone was sampled by a Monkhorst-Pack grid ${ }^{[34]}$ corresponding to $0.03 \AA^{-1}$ spacing in the reciprocal space. Starting from crystallographically determined structural data, atomic positions and cell parameters were 
relaxed, and NMR shielding calculations ${ }^{[35]}$ were performed on both relaxed and unrelaxed structures using GIPAW. ${ }^{[36]}$ The anisotropy parameters $\Delta \delta$ and $\eta$ were obtained from the absolute shielding tensor using the same convention as described in equations (14) and (15). In the surface calculations (Figure 7), the PBE exchange-correlation functional was used with the same electronic parameters as in the bulk calculations. Keeping the lattice fixed, the atoms were allowed to relax and the NMR parameters were computed on the relaxed structures.

Calculations were performed on STFC's SCARF cluster and Thomas, the UK National Tier 2 High Performance Computing Hub in Materials and Molecular Modelling.

\section{ACKNOWLEDGMENTS}

Computing resources were partially provided by STFC Scientific Computing Department's SCARF cluster. We also acknowledge support from CCC-NC funded by EPSRC (EP/M022501/1) and the UKCP consortium funded by EPSRC (EP/K013564/1). We are grateful to the UK Materials and Molecular Modelling Hub for computational resources, which is partially funded by EPSRC (EP/P020194/1).

\section{REFERENCES}

(1) (a) H. Saitô, I. Ando, A. Ramamoorthy, Prog. Nucl. Magn. Reson. Spectrosc., 57 (2010), $181-228$.

(2) (a) G. L. Hoatson, R. L. Vold, NMR Basic Principles and Progress; Springer-Verlag: Berlin, 1994; Vol. 32, pp 3-67. (b) T. M. Alam, G. P. Drobny, Chem. Rev. 91 (1991) 1545-1590. (c) A. D. Ronemus, R. R. Vold and R. L. Vold, J. Chem. Soc., Faraday Trans. 1, 84 (1988) 37613776.

(3) (a) S. L. Kohler, M. P. Klein, Biochemistry, 15 (1976) 967. (b) P. R. Collis, B. De Kruijff, Biochem. Biophys. Acta, 559 (1979) 399. (c) A. E. Aliev, K. D. M. Harris, P. H. Champkin, J. Phys. Chem. B 109 (2005) 23342-23350. 
(4) D. P. Tunstall, S. Patou, R. S. Liu, Y. H. Kao, Mater. Res. Bull. 34 (1999) 1513-1520.

(5) J. Herzfeld, A. E. Berger, J. Chem. Phys. 73 (1980) 6021-6030.

(6) HBA 1.7.5, K. Eichele, R. E. Wasylishen, Dalhousie University \& Universitat Tubingen, 2015.

(7) M. Bak, J. T. Rasmussen, N. C. Nielsen, J. Magn. Reson. 213 (2011) 366-400.

(8) T. F. Kemp, M. E. Smith, Solid State NMR 35 (2009) 243-252.

(9) A. C. Olivieri, J. Magn. Reson., Ser. A 123 (1996) 207-210.

(10) (a) L. Shao, J. Titman, Prog. Nucl. Magn. Reson. Spectrosc. 51 (2007) 103-137. (b) L. Shao, J. R. Yates, J. Titman, J. Phys. Chem. A 111 (2007) 13126-13132.

(11) (a) N. J. Clayden, C. M. Dobson, L.-Y. Lian and D. J. Smith, J. Magn. Reson. 69 (1986) 476. (b) S. E. Soss, P. F. Flynn, R. J. Iuliucci, R. P. Young, L. J. Mueller, J. Hartman, G. J. O. Beran, J. K. Harper, ChemPhysChem, 18 (2017) 1-9.

(12) P. Hodgkinson, L. Emsley, J. Chem. Phys. 107 (1997) 4808-4816.

(13) (a) G. L. Turner, K. A. Smith, R. J. Kirkpatrick, E. Oldfield, J. Magn. Reson. 70 (1986) 408415. (b) C. M. Lagier, A. C. Olivieri, D. C. Apperley, R. K. Harris, Solid State Nucl. Magn. Reson. 1 (1992) 205-210. (c) T. Nunes, A. Vainrub, M. Ribet, F. Rachdi, P. Bernier, M. Almeida, J. Chem. Phys. 96 (1992) 8021-8025. (d) K. Eichele, R. E. Wasylishen, J. Phys. Chem. 98 (1994) 3108-3113. (e) J. Sepa, R. J. Gorte, B. H. Suits, D. White, Chem. Phys. Lett. 252 (1996) 281-286. (f) N. A. Davies, R. K. Harris, A. C. Olivieri, Mol. Phys. 87 (1996) 669-677. (g) C. M. Lagier, A. C. Olivieri, J. Magn. Reson. 126 (1997) 138-141. (h) L. Eichele, A.-R. Grimmer, Can. J. Chem. 89 (2011) 870-884.

(14) N. J. Clayden, C. M. Dobson, A. Fern, J. Chem. Soc., Dalton Trans. 1989, 843-847. 
(15) C. Cossement, J. Darville, J.-M. Gilles, J. B. Nagy, C. Fernandez, J.-P. Amoureux, Magn. Reson. Chem. 30 (1992) 263-270.

(16) F. Alkan, S. T. Holmes, R. J. Iuliucci, K. T. Mueller, C. Dybowski, PCCP, 18 (2016) 1891418922.

(17) U. Haeberlen, High Resolution NMR in Solids, Selective Averaging. In Adv. Magn. Reson., Suppl., ed. J. S. Waugh, Academic Press, New York, volume 1. pp. 1-468.

(18) (a) C. Nayral, E. Viala, P. Fau, F. Senocq, J.-C. Jumas, A. Maisonnat, B. Chaudret, Chem. Eur. J. 6 (2000) 4082-4090. (b) S. Han, B. Jang, T. Kim, S. M. Oh, T. Hyeon, Adv. Funct. Mater. 15 (2005) 1845-1850. (c) Z.-W. Chen, C.-H. Shek, C. M. L. Wu, J. K. L. Lai, Front. Mater. Sci. 7 (2013) 203-226 and references therein. (d) X. Wang, L. Zhi, K. Müllen, Nano Lett. 8 (2008) 323-327. (e) J. P. Cheng, J. Wang, Q. Q. Li, H. G. Liu, Y. Li, J. Indust. Eng. Chem. 44 (2016) 1-22 and references therein; (f) G. Fedorenko, L. Oleksenko, N. Maksymovych, G. Skolyar, O. Ripko, Nanoscale Res. Lett. 12 (2017) 329; (g) C. P. Gu, Y. W. Cui, L. Y. Wang, E. H. Sheng, J. J. Shim, J. R. Huang, Sens. Actuators B Chem. 241 (2017) 298-307; (h) A. B. Kuriganova, C. A. Vlaic, S. Ivanov, D. V. Leontyeva, A. Bund, N. V. Smirnova, J. Appl. Electrochem. 46 (2016) 527-538.

(a) W. H. Bauer, A. A. Khan, Acta Cryst. B27 (1971), 2133-2139. (b) A. A. Bolzan, C. Fong, B. J. Kennedy, C. J. Howard, Acta Cryst. B53 (1997), 373-380.

(20) (a) J. P. Perdew, A. Ruzsinszky, G. I. Csonka, O. A. Vydrov, G. E. Scuseria, L. A. Constantin, X. Zhou, K. Burke, Phys. Rev. Lett. 100 (2008) 136406. (b) L. He, F. Liu, G. Hautier, M. J. T. Oliveira, M. A. L. Marques, F. D. Vila, J. J. Rehr, G.-M. Rignanese, A. Zhou, Phys. Rev. B 89 (2014) 064305. (c) P. Haas, F. Tran, P. Blaha, Phys. Rev. B, 79 (2009) 085104.] 
(21) (a) A. Bax, N. M. Szeverenyi, G. E. Maciel, J. Magn. Reson. 52 (1983) 147-152. (b) Z. Gan, J. Amer. Chem. Soc. 114 (1992) 8307-8309. (c) J. Z. Hu, W. Wang, F. Liu, M. S. Solum, D. W. Alderman, R. J. Pugmire, D. M. Grant, J. Magn. Reson. 113 (1995) 21-222. (d) H. Saitô, I. Ando, A. Ramamoorthy, Prog. Nucl. Magn. Reson. Spectrosc. 57 (2010) 181228 and references therein.

(22) A. E. Aliev, Biopolymers, 77 (2005) 230-245.

(23) (a) C. Crockford, H. Geen, J. J. Titman, Solid-state Nucl. Magn. Reson. 22 (2002) 298-310. (b) L. Shao, J. J. Titman, (2006) J. Chem. Phys., 125, 064507. (c) F. Martini, H. K. Miah, D. Iuga, M. Geppi, J. J. Titman, J. Magn. Reson, 259 (2015) 102-107.

(24) (a) M. Dračínský, P. Hodgkinson, Chem. - Eur. J. 20 (2014) 2201-2207. (b) M. Dračínský, P. Bouř, P. Hodgkinson, J. Chem. Theory Comput. 12 (2016) 968-973.

(25) J. Oviedo, M. J. Gillan, Surface Science 463 (2000), 93.

(26) R. K. Harris, T. N. Mitchell, G. J. Nesbitt, Magn. Reson. Chem. 23 (1985) 1080-1081.

(27) R. J. Wittebort, E. T. Olejniczak, R. G. Griffin, J. Chem. Phys. 86 (1987) 5411-5420.

(28) W. H. Press, B. P. Flannery, S. A. Teukolsky, W. T. Vetterling, Numerical Recipes - The Art of Scientific Computing. Cambridge University Press, Cambridge (1989).

(29) A. E. Aliev, K. D. M. Harris, Magn. Reson. Chem. 36 (1998) 855-868.

(30) M. Veshtort, R. G. Griffin, J. Magn. Reson. 178 (2006) 248-282.

(31) S. J. Clark, M. D. Segall, C. J. Pickard, P. J. Hasnip, M. J. Probert, K. Refson, M.C. Payne, Z. Kristall. 220 (2005), 567 
(32) (a) J. P. Perdew, A. Zunger, Phys. Rev. B 23 (1981), 5048. (b) J. P. Perdew, K. Burke, M. Ernzerhof, Phys. Rev. Lett. 77 (1996), 3865. (c) J. P. Perdew, Y. Wang, Phys. Rev. B 45 (1992), 13244. (d) J. P. Perdew, A. Ruzsinszky, G. I. Csonka, O. A. Vydrov, G. E. Scuseria, L. A. Constantin, X. Zhou, K. Burke, Phys. Rev. Lett. 100 (2008), 136406.

(33) J. Autschbach, T. J. Ziegler, Chem. Phys. 113 (2000), 9410.

(34) H. J. Monkhorst, J. D. Pack, Phys. Rev. B 13 (1976), 5188.

(35) C. Bonhomme, C. Gervais, F. Babonneau, C. Coelho, F. Pourpoint, T. Azais, S. E. Ashbrook, J. M. Griffin, J. R. Yates, F. Mauri, C. J. Pickard, Chem. Rev. 112 (2012), 5733.

(36) (a) C. J. Pickard, F. Mauri, Phys. Rev. B 63 (2001), 245101. (b) J. R. Yates, C. J. Pickard, F. Mauri, Phys. Rev. B 76 (2007), 024401. 


\title{
Supporting Information
}

\section{Tin Chemical Shift Anisotropy in Tin Dioxide: On Ambiguity of CSA Asymmetry Derived from MAS Spectra}

\author{
Abil E. Aliev, ${ }^{a *}$ Albert P. Bartók, ${ }^{b}$ Jonathan R. Yates ${ }^{c}$
}

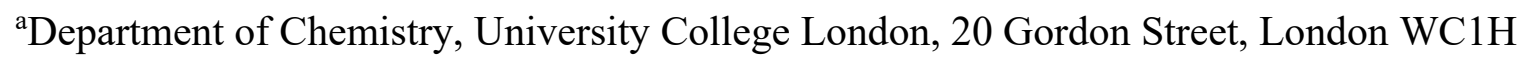
0AJ, U.K.

bscientific Computing Department, Rutherford Appleton Laboratory, Harwell Campus, Didcot OX11 0QX, UK

${ }^{\mathrm{c}}$ Department of Materials, University of Oxford, Parks Road, Oxford OX1 3PH, UK 


\section{Dependence of the error function on the value of the asymmetry parameter}

In order to investigate the sensitivity of the error function to the value of the asymmetry parameter, $\eta$, we have carried out additional iterative fittings at fixed values of $\eta$ to determine the corresponding value of $s$ dif (equation (4) in the main text). As shown in Figure S1, only very small changes in $s$ dif are observed for $\eta$ values between 0 and 0.02 , suggesting that the error function is considerably less sensitive to the asymmetry parameter in this range compared to $\eta$ values greater than 0.02 . Nevertheless, the minimum in the $s$ dif value is well-defined (Figure S1b) without any other local minima and the best-fit value of the asymmetry parameter at $0.0 \pm 0.011$ was reproducible when using experimental spectra processed differently or when using a different program (Table 1 in the main text, $3^{\text {rd }}$ column).

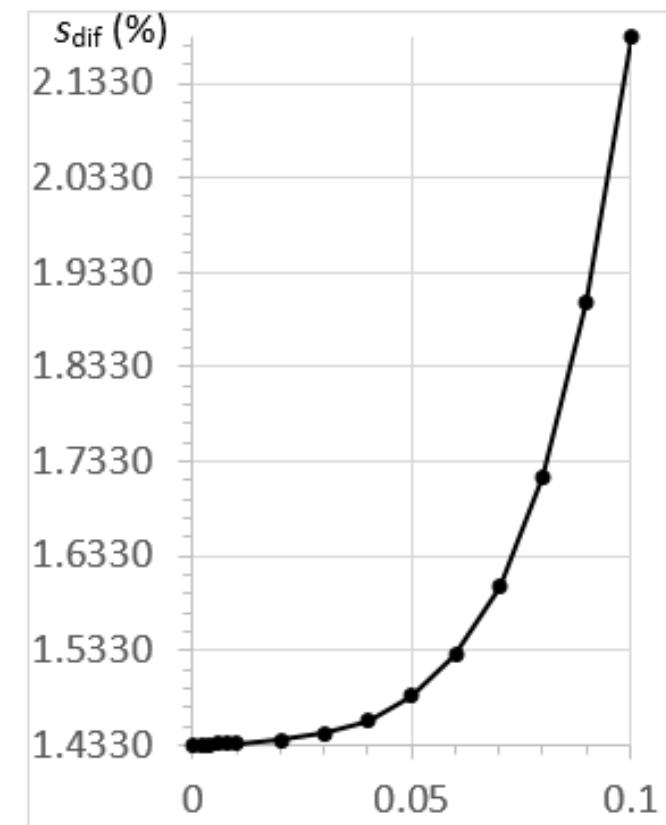

Asymmetry parameter, $\eta$

(a)

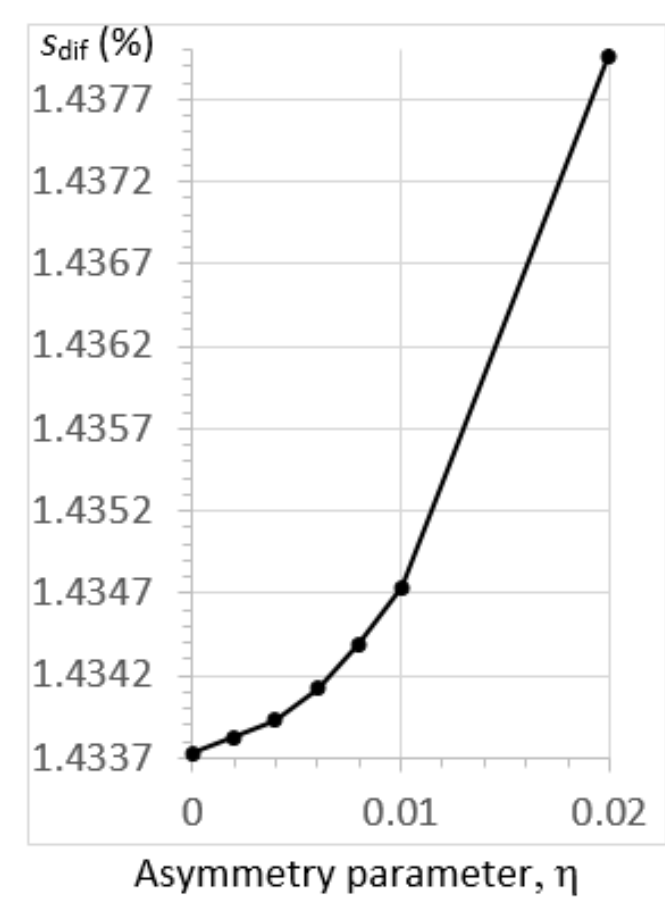

(b)

Figure S1. (a) The dependence of the error function $s_{\text {dif }}$ (in \%) on the CSA asymmetry parameter, $\eta$; (b) The expanded view of the dependence shown in (a) near the minimim value of $s$ dif. 


\section{Supplementary figures showing simulated and experimental MAS spectra}

(c)

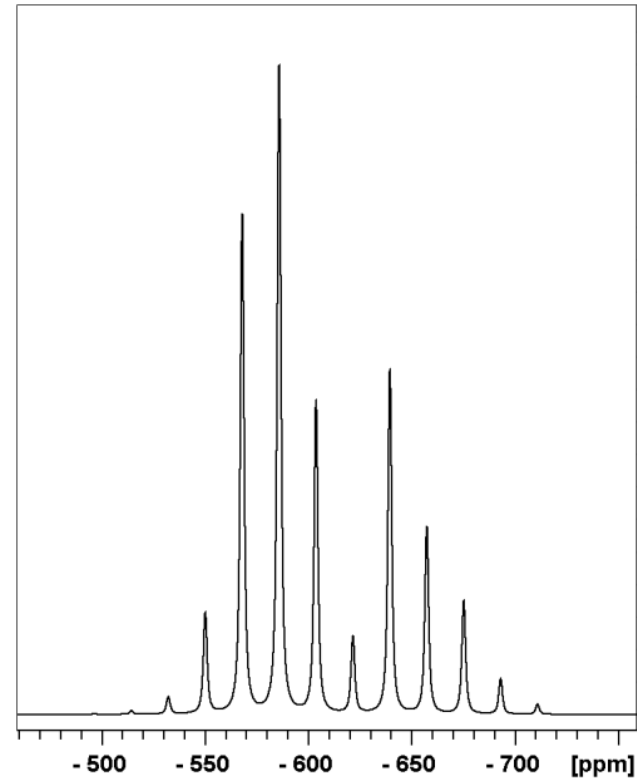

(a)

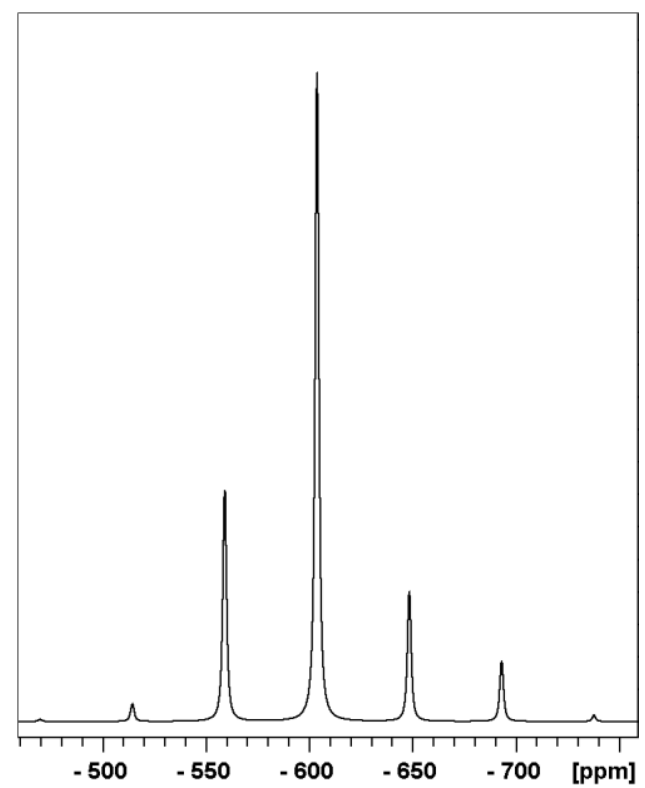

(d)

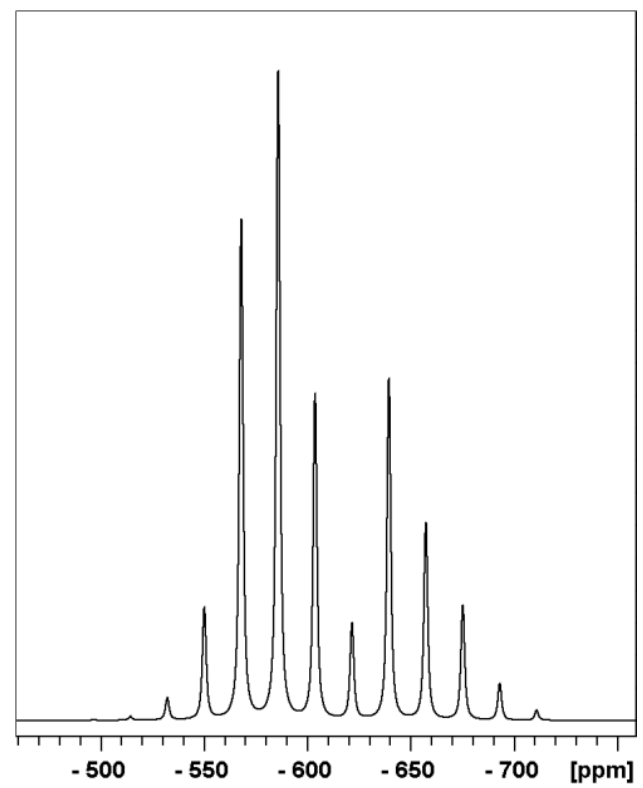

(b)

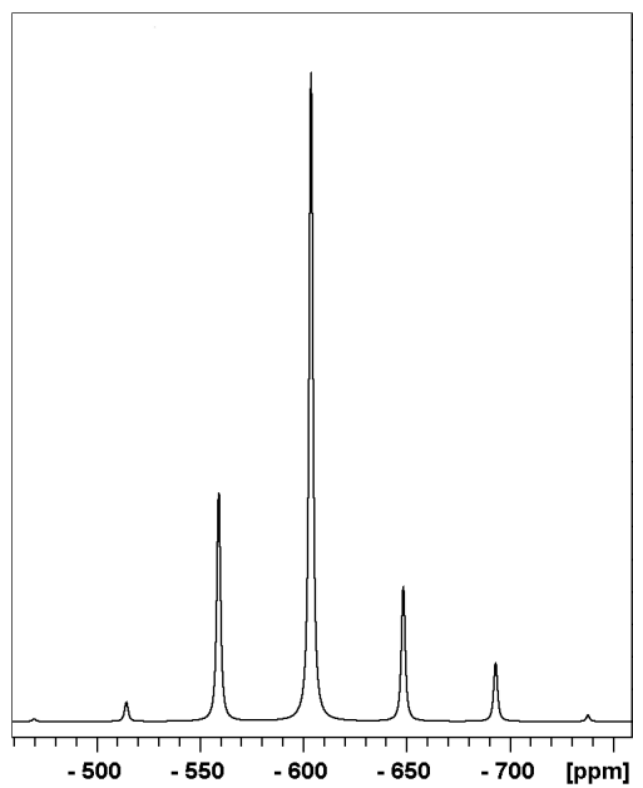

Figure S2. The iteratively fitted spectra at MAS frequencies of $5 \mathrm{kHz}$ ( $\mathrm{a}$ and b) and $2 \mathrm{kHz}(\mathrm{c}$ and d), using (a) $\Delta \delta=-127.70 \mathrm{ppm}$ and $\eta=0.0$; (b) $\Delta \delta=-126.29 \mathrm{ppm}$ and $\eta=0.285$; (c) $\Delta \delta=$ $-125.62 \mathrm{ppm}$ and $\eta=0$ and (d) $\Delta \delta=-125.24 \mathrm{ppm}$ and $\eta=0.179$. The overlaid view of spectra is shown in Figure S3. 

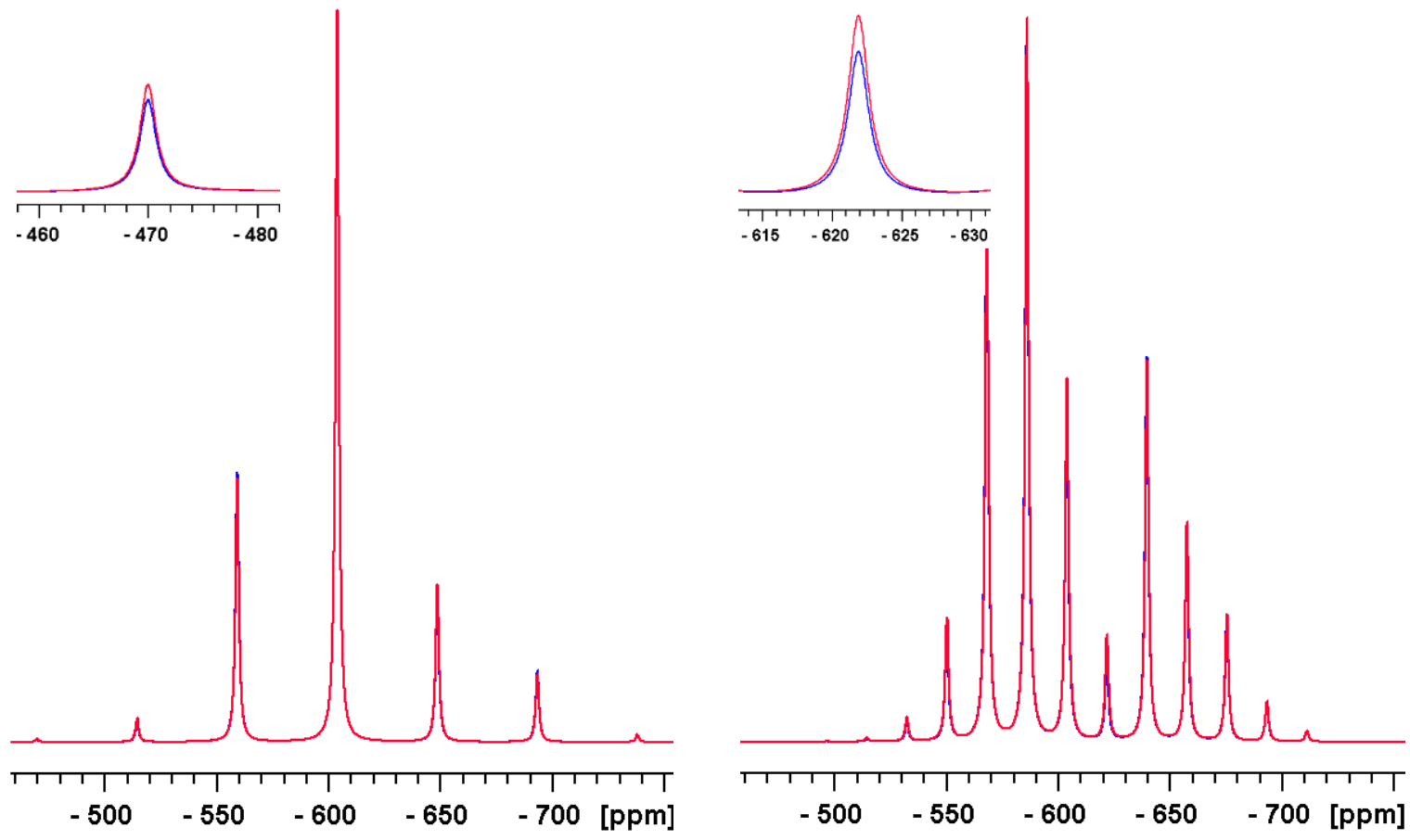

Figure S3. The iteratively fitted spectra at MAS frequencies of $5 \mathrm{kHz}$ (a) and $2 \mathrm{kHz}$ (b), using (a) $\Delta \delta=-127.70 \mathrm{ppm} / \eta=0.0$ (in red) and $\Delta \delta=-126.29 \mathrm{ppm} / \eta=0.285$ (in blue); (c) $\Delta \delta=-125.62 \mathrm{ppm} / \eta=0$ (in red) and $\Delta \delta=-125.24 \mathrm{ppm} / \eta=0.179$ (in blue). In both cases, the spectrum simulated with $\eta=0$ (in red) was overlaid over that with $\eta \neq 0$ (in blue). Relatively large differences are observed at $\sim-470 \mathrm{ppm}$ (+3 spinning sideband) in (a) and at $\sim-622 \mathrm{ppm}$ (-1 spinning sideband) in (b), expanded views of which are shown above the main figures. 


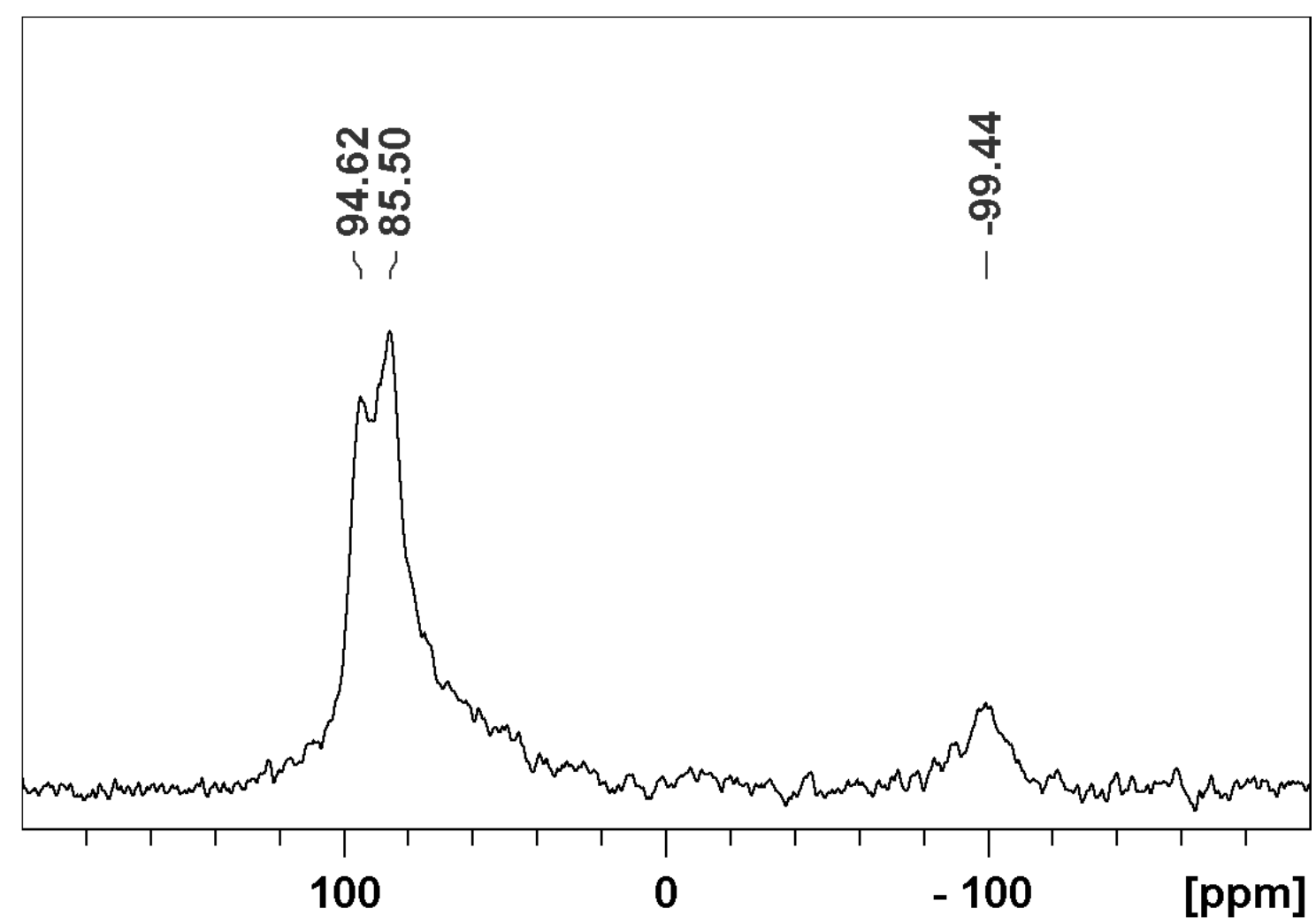

Figure S4. The solid-state single-pulse ${ }^{31} \mathrm{P}$ NMR spectrum (121.5 MHz, 7.05 T) with proton decoupling of triphenyl phospine oxide ( $\mathrm{TPPO}, \mathrm{Ph}_{3} \mathrm{PO}$ ) recorded without sample spinning using an hour long recycle delay for 16 hours, a $30^{\circ}$ pulse (with a duration $0.77 \mu \mathrm{s}$ ) and a receiver dead time of $6.5 \mu \mathrm{s}$. The spectrum shows severe distortions of the ${ }^{31} \mathrm{P}$ lineshape with a reduced intensity between the low- and high-frequency edges of the spectrum at 94.6 and $-99.4 \mathrm{ppm}$. Nevertheless, the distortion of the lineshape shows clearly that the CSA responsible for the ${ }^{31} \mathrm{P}$ static lineshape is not axially symmetric, with the $\delta_{11}$ and $\delta_{22}$ components resolved at 94.6 and $85.5 \mathrm{ppm}$. The ${ }^{31} \mathrm{P}$ chemical shifts are given relative to $85 \%$ aqueous solution of $\mathrm{H}_{3} \mathrm{PO}_{4}(0 \mathrm{ppm})$. We also attempted to distort the lineshape in the ${ }^{119} \mathrm{Sn}$ SSNMR spectrum of tin dioxide by reducing the dead time, the recycle delay in signle-pulse experiments, by over left-shifting the Hahn echo and changing the field (see Figure S6) in order to resolve a possible difference in the $\delta_{11}$ and $\delta_{22}$ components. However, in none of the cases such a resolution was achieved or asymmetry in the high-frequency region was observed. 


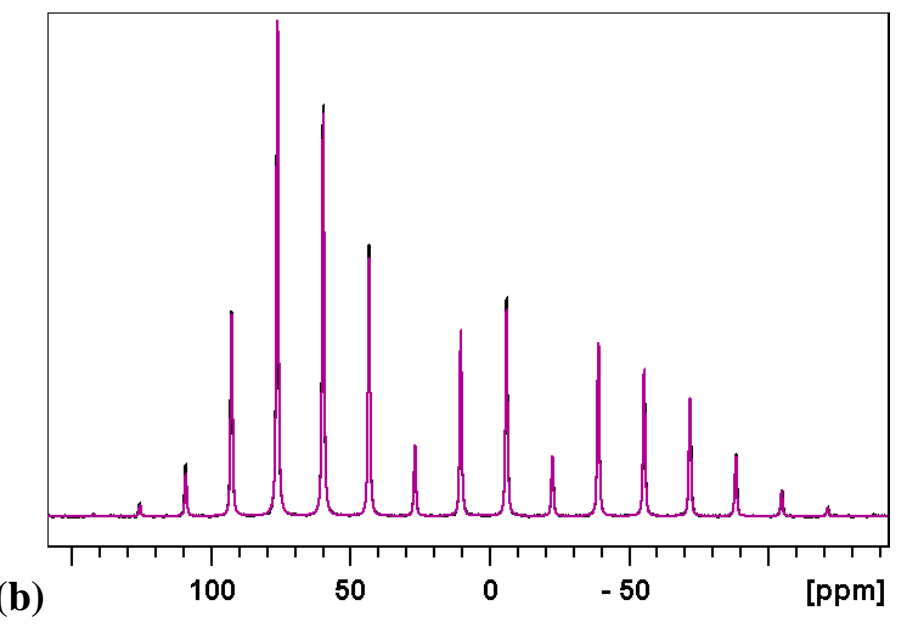

(b)

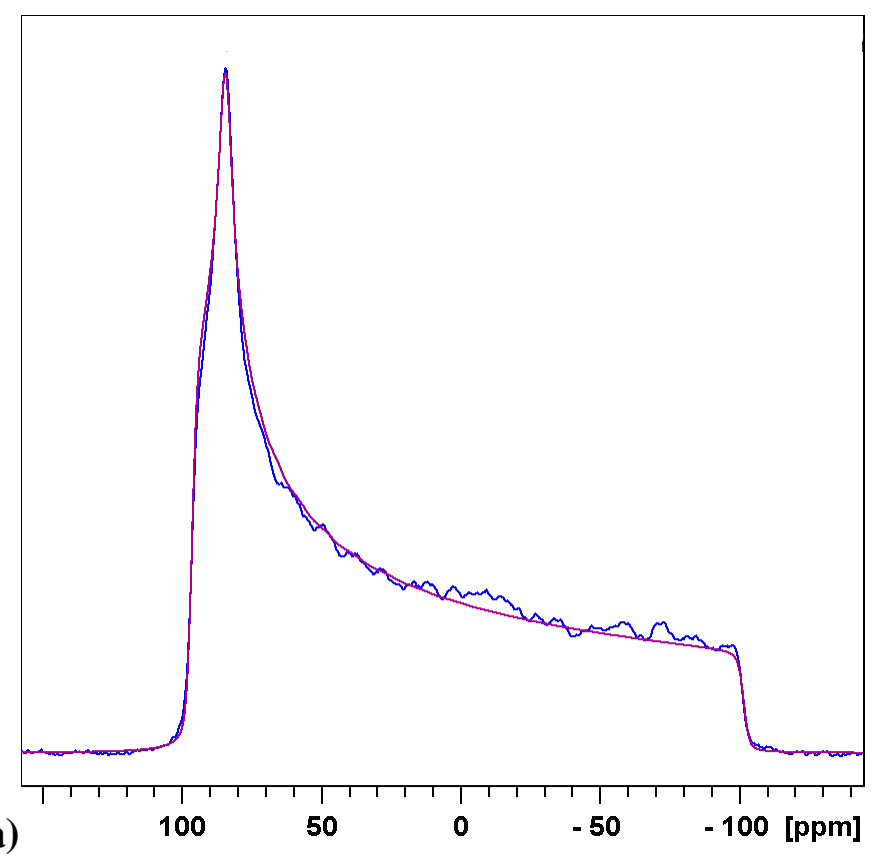

Figure S5. The experimental ${ }^{31} \mathrm{P}$ CP NMR spectra (shown in black) of the monoclinic polymorph of TPPO recorded without sample spinning (a) and at MAS frequency of $2 \mathrm{kHz}$, overlaid with the calculated spectra (shown in purple) with the best-fit values of $\Delta \delta=-192.1 \mathrm{ppm} / \eta=0.094$ in (a) and $\Delta \delta=-189.3 \mathrm{ppm} / \eta=0.041$ in (b). The previously reported values of $\Delta \delta / \eta$ were $-189.8 \mathrm{ppm} / 0.075[\mathrm{I}$. G. Shenderovich, J. Phys. Chem. C 117 (2013) 26689-26702]. The axial asymmetry of the ${ }^{31}$ P CSA is apparent from the lineshape of the static powder pattern between 80 and $100 \mathrm{ppm}$. The linewidth of the isotropic peak in the MAS spectrum is $73 \pm 1 \mathrm{~Hz}$. Acquisition conditions for ${ }^{31} \mathrm{P} \mathrm{CP}$ experiments were: ${ }^{1} \mathrm{H} 90^{\circ}$ pulse duration $=2.45 \mu \mathrm{s}$; contact time $=2 \mathrm{~ms}$; recycle delay $=900 \mathrm{~s}$. Note that the closest ${ }^{31} \mathrm{P}-$ ${ }^{31} \mathrm{P}$ distance is $7.429 \AA$ in the monoclinic polymorph of TPPO [Brock, C. P.; Schweizer, W. B.; Dunitz, J. D. J. Am. Chem. Soc. 107 (1985) 6964-6970], significantly smaller than that in ammonium dihydrogen phosphate (ADP, $4.2 \AA$ [K. Eichele, R. E. Wasylishen, J. Phys. Chem. 98 (1994) 3108$3113]$ ), thus the ${ }^{31} \mathrm{P}_{-}{ }^{31} \mathrm{P}$ dipolar coupling is only $48 \mathrm{~Hz}$ (270 Hz in ADP). 


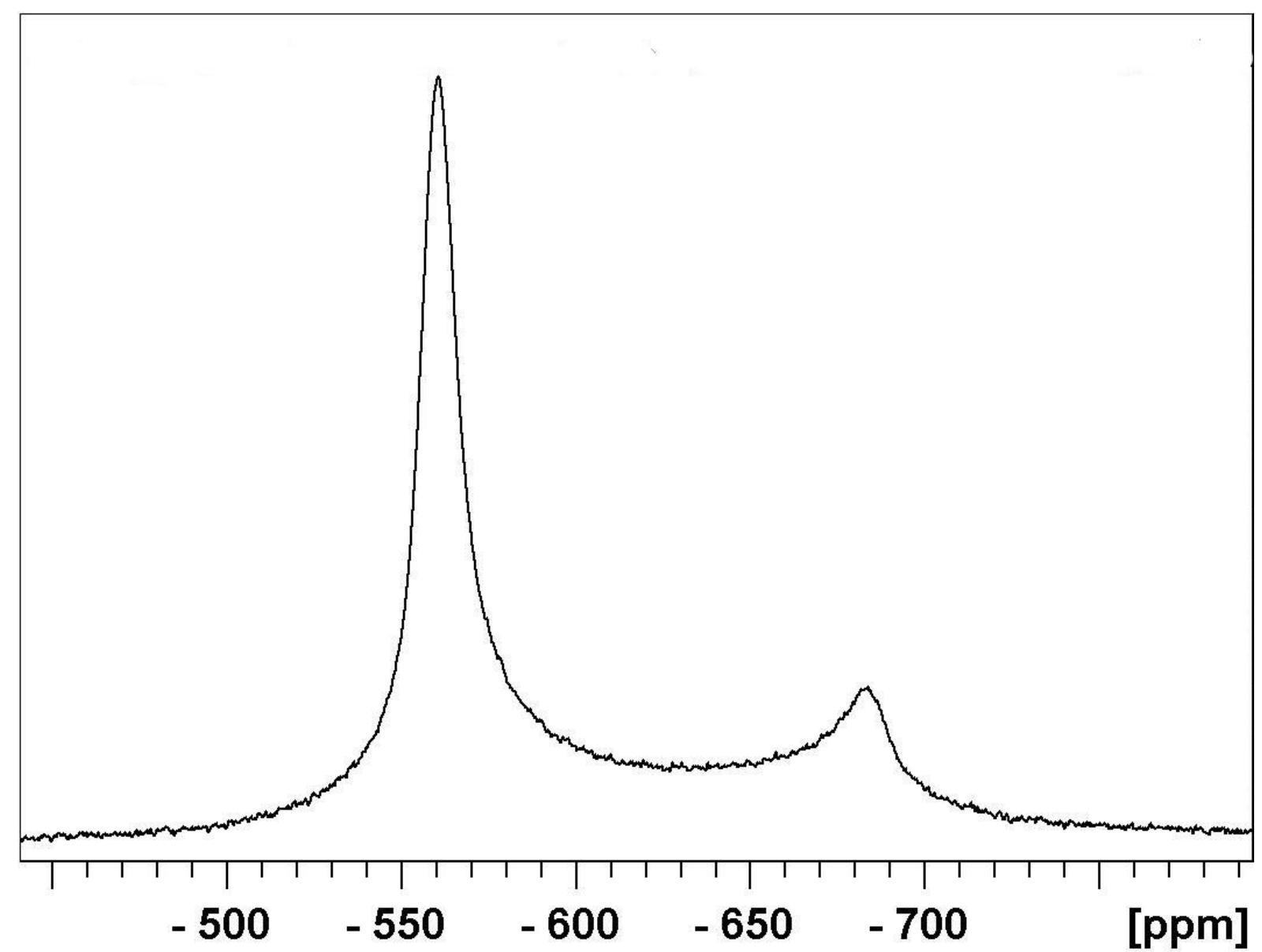

Figure S6. The single-pulse ${ }^{119} \mathrm{Sn}$ NMR spectrum $(261.2 \mathrm{MHz}, 16.44 \mathrm{~T})$ recorded without sample spinning using a solution NMR instrument, Bruker Avance Neo 700. A recycle delay of $2 \mathrm{~s}$, a receiver dead time of $6.5 \mu$ s and a $15^{\circ}$ pulse (with a duration $1.87 \mu \mathrm{s}$ ) were used. The spectrum shows distortions of the ${ }^{119} \mathrm{Sn}$ lineshape with a reduced intensity between the low- and high-frequency edges of the spectrum at -560 and $-685 \mathrm{ppm}$. Unlike the lineshape shown in Figure S4 corresponding to $\eta=$ 0.09 , no slitting is observed in the high-frequency region of the spectrum, as expected for the axially symmetric lineshape with $\eta=0$. 


\section{Supplementary tables with the results of the analysis of static and MAS spectra}

Table S1. The values of ${ }^{119}$ Sn CSA parameters determined from the Herzfeld-Berger analysis of spinning sideband intensities in MAS spectra (frequency shown in $\mathrm{kHz}$ ), using two different starting values of $\eta, 0.0$ and 0.5 . The fixed values of isotropic chemical shifts from lineshape fittings (Table 1) were used.

\begin{tabular}{|c|c|c|c|c|}
\hline Parameter & $2 \mathrm{kHz}$ & $2 \mathrm{kHz}$ & $5 \mathrm{kHz}$ & $5 \mathrm{kHz}$ \\
\hline$\delta_{\text {iso, }}, \mathrm{ppm}$ & -603.81 & -603.81 & -603.79 & -603.79 \\
\hline$\Delta \delta, \mathrm{ppm}$ & $-127.8 \pm 0.7$ & $-126.4 \pm 1.1$ & $-129.0 \pm 0.8$ & $-126.2 \pm 0.8$ \\
\hline$\eta$ & $0.000 \pm 0.013$ & $0.274 \pm 0.014^{a}$ & $0.000 \pm 0.009$ & $0.413 \pm 0.011^{a}$ \\
\hline$\delta_{11}, \mathrm{ppm}$ & $-561.2 \pm 0.9$ & $-550.1 \pm 1.0$ & $-560.8 \pm 0.7$ & $-544.4 \pm 0.8$ \\
\hline$\delta_{22}, \mathrm{ppm}$ & $-561.2 \pm 0.6$ & $-573.2 \pm 0.7$ & $-560.8 \pm 0.4$ & $-579.1 \pm 0.5$ \\
\hline$\delta_{33}, \mathrm{ppm}$ & $-689.0 \pm 0.7$ & $-688.1 \pm 0.8$ & $-689.8 \pm 0.5$ & $-688.0 \pm 0.6$ \\
\hline$\Omega, \mathrm{ppm}$ & $127.8 \pm 0.8$ & $138.0 \pm 0.8$ & $129.0 \pm 0.6$ & $143.6 \pm 0.5$ \\
\hline$\mu$ & 7.153 & 7.721 & 2.887 & 3.214 \\
\hline$\kappa$ & $1.000 \pm 0.013$ & $0.6665 \pm 0.014$ & $1.000 \pm 0.009$ & $0.516 \pm 0.010$ \\
\hline$s_{\text {cond }}(\mu)$ & 0.032 & 0.026 & 0.014 & 0.008 \\
\hline$s_{\operatorname{marg}}(\mu)$ & 0.044 & 0.044 & 0.014 & 0.012 \\
\hline$R(\mu)$ & 1.89 & 2.86 & 1.00 & 2.25 \\
\hline$S_{\text {cond }}(\kappa)$ & 0.009 & 0.008 & 0.009 & 0.007 \\
\hline$s_{\operatorname{marg}}(\kappa)$ & 0.013 & 0.014 & 0.009 & 0.010 \\
\hline$R(\kappa)$ & 2.09 & 3.06 & 1.00 & 2.04 \\
\hline$s_{\mathrm{fit}}\left(\times 10^{-3}\right)$ & 5.956 & 2.982 & 3.755 & 1.902 \\
\hline
\end{tabular}

${ }^{a}$ In terms of their selectivity in favouring the correct CSA asymmetry parameter in tin dioxide, the full lineshape analysis (Table 1) performs relatively better than the analysis of spinning sideband intensities presented in this table. This is evident from the best-fit $\eta$ values determined at the MAS frequency of $5 \mathrm{kHz}$ : 0.29 from the lineshape fittings and 0.41 from the analysis of spinning sideband intensities. The reason for better performance could be due to the very large number of points fitted in the lineshape analysis. Nevertheless, the example of $\mathrm{SnO}_{2}$ shows that both the MAS lineshape and spinning sideband analyses may overestimate the $\eta$ value by as much as $\sim 0.3$ and $\sim 0.4$, respectively, at the extremums of the error functions used. 
Table S2. The values of ${ }^{31} \mathrm{P}$ CSA parameters triphenylphosphine oxide determined from the analysis of static and MAS (frequency shown in $\mathrm{kHz}$ ) lineshapes, using the Bruker curve fitting program SoLA (Solid Lineshape Analysis, version 2.2.4). The chemical shift range between $+220 \mathrm{ppm}$ and $-220 \mathrm{ppm}$ was selected for fittings. All the calculated static and MAS lineshapes were convoluted by Lorentzian and Gaussian line-broadening functions with the linewidth parameters of $L B$ and $G B$, respectively. Typical values of $L B / G B$ were $133 / 337 \mathrm{~Hz}$ in fittings of the static lineshape and $60 / 65 \mathrm{~Hz}$ in fittings of MAS spectra.

\begin{tabular}{rrrrrrrr}
\hline Parameter & Static & $\mathbf{2 ~ k H z}$ & $\mathbf{3 ~ k H z}$ & $\mathbf{4 ~ k H z}$ & $\mathbf{5 ~ k H z}$ & $\mathbf{6 ~ k H z}$ & $\mathbf{7 ~ k H z}$ \\
\hline$\delta_{\text {iso }}, \mathrm{ppm}$ & 26.66 & 26.94 & 26.94 & 26.97 & 26.95 & 26.94 & 26.97 \\
$\Delta \delta, \mathrm{ppm}$ & -192.10 & -189.28 & -188.48 & -190.82 & -190.50 & -189.20 & -189.89 \\
$\eta$ & 0.094 & 0.041 & 0.125 & 0.001 & 0.121 & 0.032 & 0.102 \\
$\delta_{11}, \mathrm{ppm}$ & 96.74 & 92.62 & 97.60 & 90.66 & 98.15 & 92.01 & 96.73 \\
$\delta_{22}, \mathrm{ppm}$ & 84.65 & 87.43 & 81.93 & 90.50 & 82.94 & 88.01 & 83.80 \\
$\delta_{33}, \mathrm{ppm}$ & -101.41 & -99.25 & -98.71 & -100.24 & -100.05 & -99.19 & -99.62 \\
$\Omega, \mathrm{ppm}$ & 198.16 & 191.87 & 196.30 & 190.90 & 198.20 & 191.21 & 196.35 \\
$\kappa$ & 0.88 & 0.95 & 0.84 & 1.00 & 0.84 & 0.96 & 0.87 \\
\hline Best overlap, \% & 97.6 & 94.3 & 93.7 & 93.5 & 93.8 & 94.5 & 94.6 \\
\hline
\end{tabular}


Table S3. The values of ${ }^{31} \mathrm{P}$ CSA parameters determined from the Herzfeld-Berger analysis of MAS spectra (frequency shown in $\mathrm{kHz}$ ), using the HBA program (version 1.7.5). The isotropic chemical shift values from full lineshape fittings (Table S2) are used.

\begin{tabular}{rrrrrrr}
\hline Parameter & $\mathbf{2 ~ k H z}$ & $\mathbf{2 ~ k H z}$ & $\mathbf{3 ~ k H z}$ & $\mathbf{3 ~ k H z}$ & $\mathbf{4 ~ k H z}$ & $\mathbf{4 ~ k H z}$ \\
\hline$\delta_{\text {iso }}, \mathrm{ppm}$ & 26.94 & 26.94 & 26.94 & 26.94 & 26.97 & 26.97 \\
$\Delta \delta, \mathrm{ppm}$ & $-188.3 \pm 3.1$ & $-188.3 \pm 0.9$ & $-187.9 \pm 2.2$ & $-188.2 \pm 0.9$ & $-190.2 \pm 1.3$ & $-190.1 \pm 1.0$ \\
$\eta$ & $0.094 \pm 0.027$ & $0.000 \pm 0.008$ & $0.085 \pm 0.019$ & $0.000 \pm 0.008$ & $0.010 \pm 0.010$ & $0.000 \pm 0.010$ \\
$\delta_{11}, \mathrm{ppm}$ & $95.6 \pm 2.8$ & $89.7 \pm 0.8$ & $94.9 \pm 2.0$ & $89.7 \pm 0.7$ & $91.0 \pm 1.1$ & $90.3 \pm 1.1$ \\
$\delta_{22}, \mathrm{ppm}$ & $83.8 \pm 1.9$ & $89.7 \pm 0.5$ & $84.2 \pm 1.3$ & $89.7 \pm 0.5$ & $89.8 \pm 0.7$ & $90.3 \pm 0.7$ \\
$\delta_{33}, \mathrm{ppm}$ & $-98.6 \pm 1.1$ & $-98.6 \pm 0.6$ & $-98.3 \pm 1.5$ & $-98.5 \pm 0.6$ & $-99.8 \pm 0.8$ & $-99.8 \pm 0.8$ \\
$\Omega, \mathrm{ppm}$ & $194.2 \pm 1.7$ & $188.3 \pm 0.5$ & $193.3 \pm 1.3$ & $188.2 \pm 0.5$ & $190.8 \pm 1.0$ & $190.1 \pm 1.0$ \\
$\kappa$ & $0.878 \pm 0.028$ & $1.000 \pm 0.008$ & $0.889 \pm 0.020$ & $1.000 \pm 0.008$ & $0.987 \pm 0.010$ & $1.000 \pm 0.009$ \\
$R(\mu)$ & 14.33 & 1.08 & 6.86 & 1.00 & 1.15 & 1.15 \\
$R(\kappa)$ & 16.00 & 1.00 & 6.25 & 1.00 & 1.23 & 1.00 \\
\hline$S_{\text {fit }}\left(\times 10^{-3}\right)$ & 2.146 & 2.330 & 2.290 & 2.374 & 3.750 & 3.743 \\
\hline
\end{tabular}

\begin{tabular}{rrrrrrr}
\hline Parameter & $\mathbf{5 ~ k H z}$ & $\mathbf{5 ~ k H z}$ & $\mathbf{6 ~ k H z}$ & $\mathbf{6 ~ k H z}$ & $\mathbf{7 ~ k H z}$ & $\mathbf{7 ~ k H z}$ \\
\hline$\delta_{\text {iso }}, \mathrm{ppm}$ & 26.95 & 26.95 & 26.94 & 26.94 & 26.97 & 26.97 \\
$\Delta \delta, \mathrm{ppm}$ & $-189.4 \pm 1.0$ & $-187.7 \pm 1.0$ & $-187.7 \pm 1.7$ & $-187.7 \pm 1.0$ & $-188.5 \pm 1.0$ & $-188.5 \pm 1.0$ \\
$\eta$ & $0.025 \pm 0.010$ & $0.000 \pm 0.010$ & $0.042 \pm 0.014$ & $0.000 \pm 0.008$ & $0.020 \pm 0.008$ & $0.000 \pm 0.008$ \\
$\delta_{11}, \mathrm{ppm}$ & $91.1 \pm 1.0$ & $89.5 \pm 1.0$ & $92.1 \pm 1.5$ & $89.5 \pm 0.9$ & $91.0 \pm 0.9$ & $89.8 \pm 0.9$ \\
$\delta_{22}, \mathrm{ppm}$ & $88.0 \pm 0.6$ & $89.5 \pm 0.6$ & $86.9 \pm 0.9$ & $89.5 \pm 0.6$ & $88.6 \pm 0.6$ & $89.8 \pm 0.6$ \\
$\delta_{33}, \mathrm{ppm}$ & $-98.3 \pm 0.8$ & $-98.2 \pm 0.8$ & $-98.3 \pm 1.1$ & $-98.3 \pm 0.7$ & $-98.7 \pm 0.7$ & $-98.7 \pm 0.7$ \\
$\Omega, \mathrm{ppm}$ & $189.4 \pm 1.0$ & $187.7 \pm 1.0$ & $190.4 \pm 1.2$ & $187.8 \pm 0.8$ & $189.7 \pm 0.7$ & $188.5 \pm 0.7$ \\
$\kappa$ & $0.967 \pm 0.009$ & $1.000 \pm 0.009$ & $0.945 \pm 0.013$ & $1.000 \pm 0.008$ & $0.974 \pm 0.020$ & $1.000 \pm 0.008$ \\
$R(\mu)$ & 1.09 & 1.09 & 2.78 & 1.00 & 1.00 & 1.00 \\
$R(\kappa)$ & 1.00 & 1.00 & 2.64 & 1.00 & 1.00 & 1.00 \\
\hline$S_{\text {fit }}\left(\times 10^{-3}\right)$ & 4.100 & 4.087 & 3.267 & 3.245 & 2.801 & 2.794 \\
\hline & & & & & &
\end{tabular}




\section{CIF files used in DFT calculations}

Structure (I) in Table 3, from reference [19a] W. H. Bauer, A. A. Khan, Acta Cryst. B27 (1971), 2133-2139:

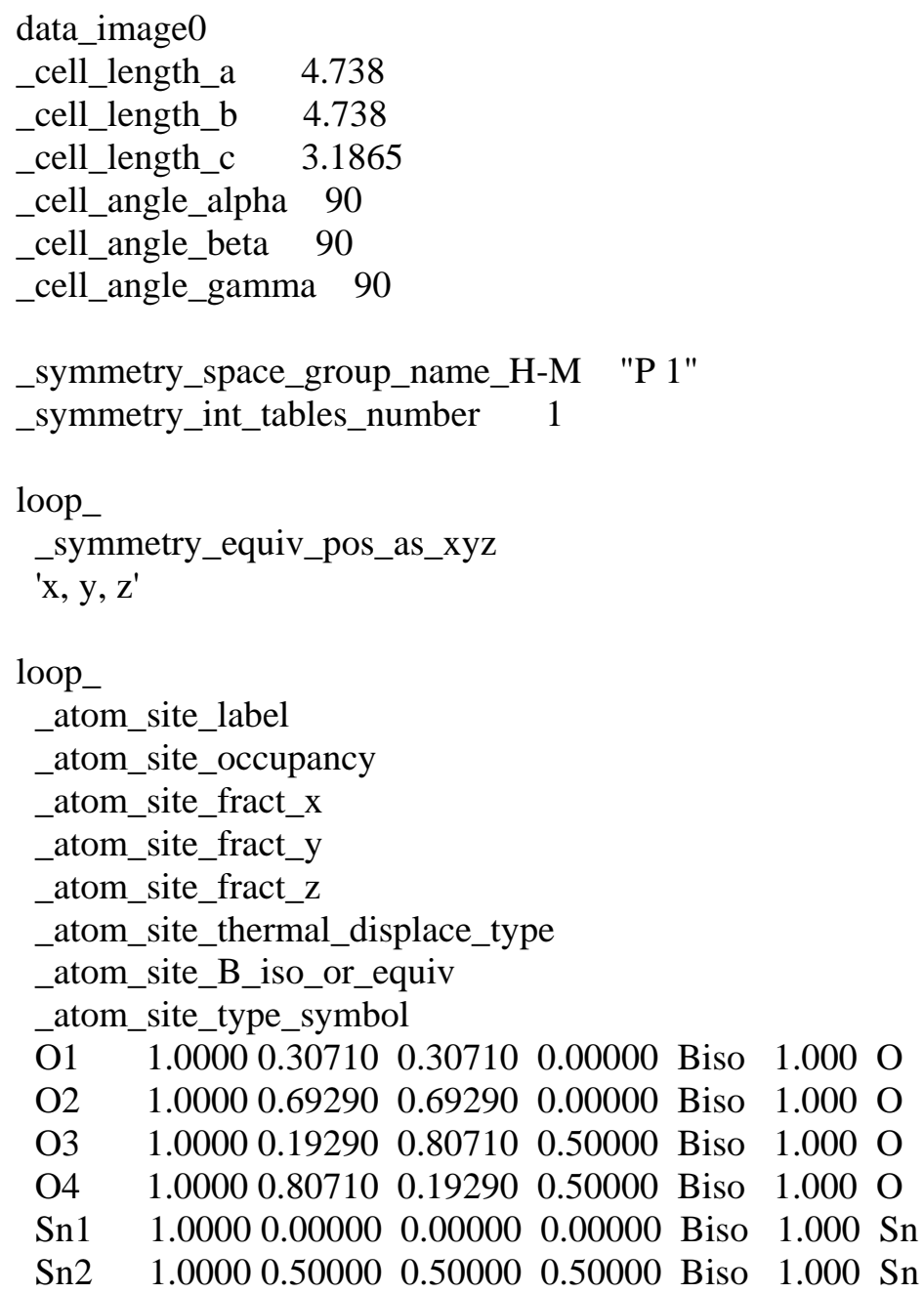

Structure (II) in Table 3, from reference [19b] A. A. Bolzan, C. Fong, B. J. Kennedy, C. J. Howard, Acta Cryst. B53 (1997), 373-380:

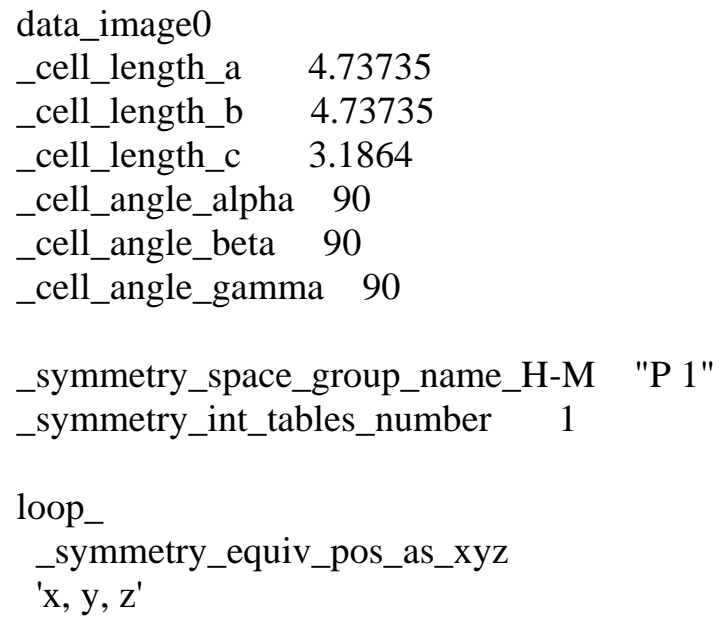




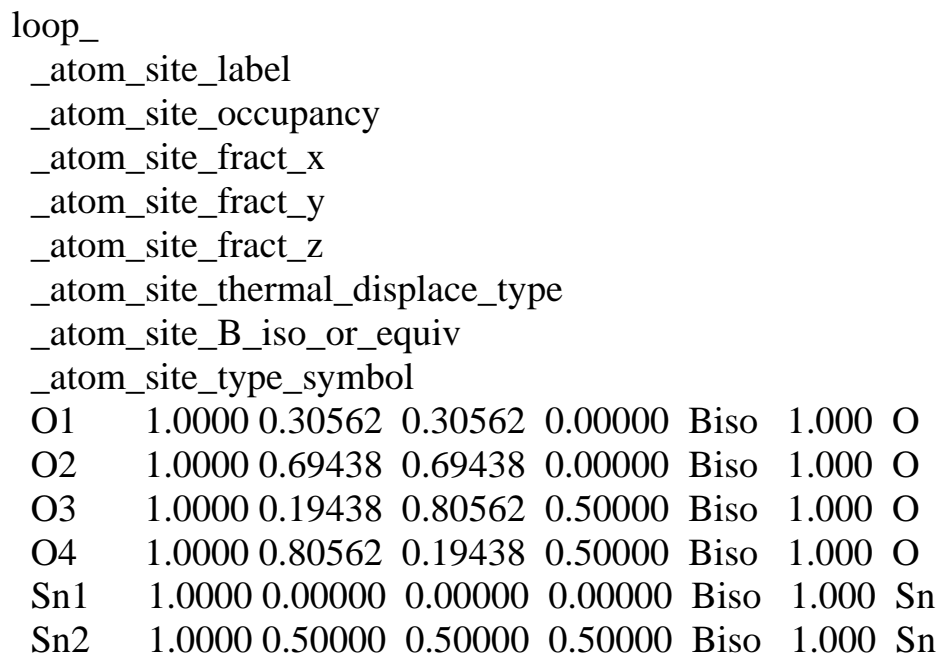

\section{LDA optimised structure (Tables 2 and 3):}

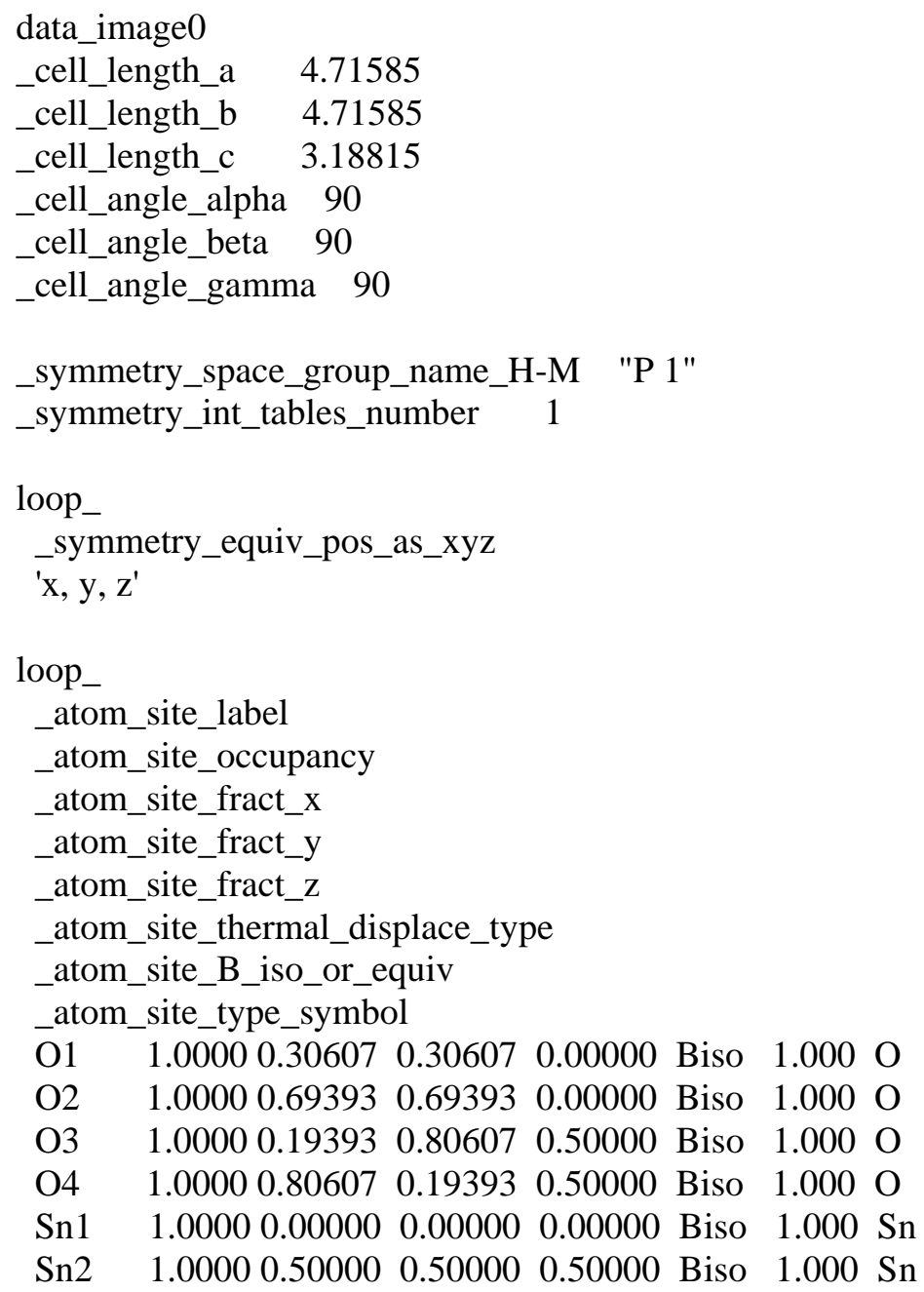

\section{PBE optimised structure (Tables 2 and 3):}

data_image 0

_cell_length_a 4.81865 
_cell_length_b 4.81865

_cell_length_c 3.23355

_cell_angle_alpha 90

_cell_angle_beta 90

_cell_angle_gamma 90

_symmetry_space_group_name_H-M "P 1"

_symmetry_int_tables_number

loop_

_symmetry_equiv_pos_as_xyz

'x, y, z'

loop

_atom_site_label

_atom_site_occupancy

_atom_site_fract_x

_atom_site_fract_y

_atom_site_fract_z

_atom_site_thermal_displace_type

_atom_site_B_iso_or_equiv

_atom_site_type_symbol

O1 $\quad \begin{array}{lllllll}1.0000 & 0.30659 & 0.30659 & 0.00000 & \text { Biso } & 1.000 \mathrm{O}\end{array}$

$\begin{array}{lllllllll}\mathrm{O} 2 & 1.0000 & 0.69341 & 0.69341 & 0.00000 & \text { Biso } & 1.000 \mathrm{O}\end{array}$

$\begin{array}{llllllllll}\mathrm{O} 3 & 1.0000 & 0.19341 & 0.80659 & 0.50000 & \text { Biso } & 1.000 \mathrm{O}\end{array}$

O4 1.00000 .806590 .193410 .50000 Biso $1.000 \mathrm{O}$

Sn1 $1.00000 .00000 \quad 0.000000 .00000$ Biso $1.000 \mathrm{Sn}$

Sn2 1.00000 .500000 .500000 .50000 Biso $1.000 \mathrm{Sn}$

\section{PW91 optimised structure (Tables 2 and 3):}

data_image 0

_cell_length_a 4.81362

_cell_length_b 4.81362

_cell_length_c 3.23211

_cell_angle_alpha 90

_cell_angle_beta 90

_cell_angle_gamma 90

_symmetry_space_group_name_H-M "P 1"

_symmetry_int_tables_number 1

loop_

_symmetry_equiv_pos_as_xyz

'x, y, z'

loop_

_atom_site_label

_atom_site_occupancy

_atom_site_fract_x

_atom_site_fract_y

_atom_site_fract_z

_atom_site_thermal_displace_type

_atom_site_B_iso_or_equiv

_atom_site_type_symbol 
$\begin{array}{lllllllll}\text { O1 } & 1.0000 & 0.30651 & 0.30651 & 0.00000 & \text { Biso } & 1.000 \mathrm{O}\end{array}$

O2 1.00000 .693490 .693490 .00000 Biso $1.000 \mathrm{O}$

O3 1.00000 .193490 .806510 .50000 Biso $1.000 \mathrm{O}$

$\begin{array}{llllllllll}\text { O4 } & 1.0000 & 0.80651 & 0.19349 & 0.50000 & \text { Biso } & 1.000 \mathrm{O}\end{array}$

Sn1 1.00000 .000000 .000000 .00000 Biso $1.000 \mathrm{Sn}$

Sn2 1.00000 .500000 .500000 .50000 Biso $1.000 \mathrm{Sn}$

\section{PBEsol optimised structure (Tables 2 and 3):}

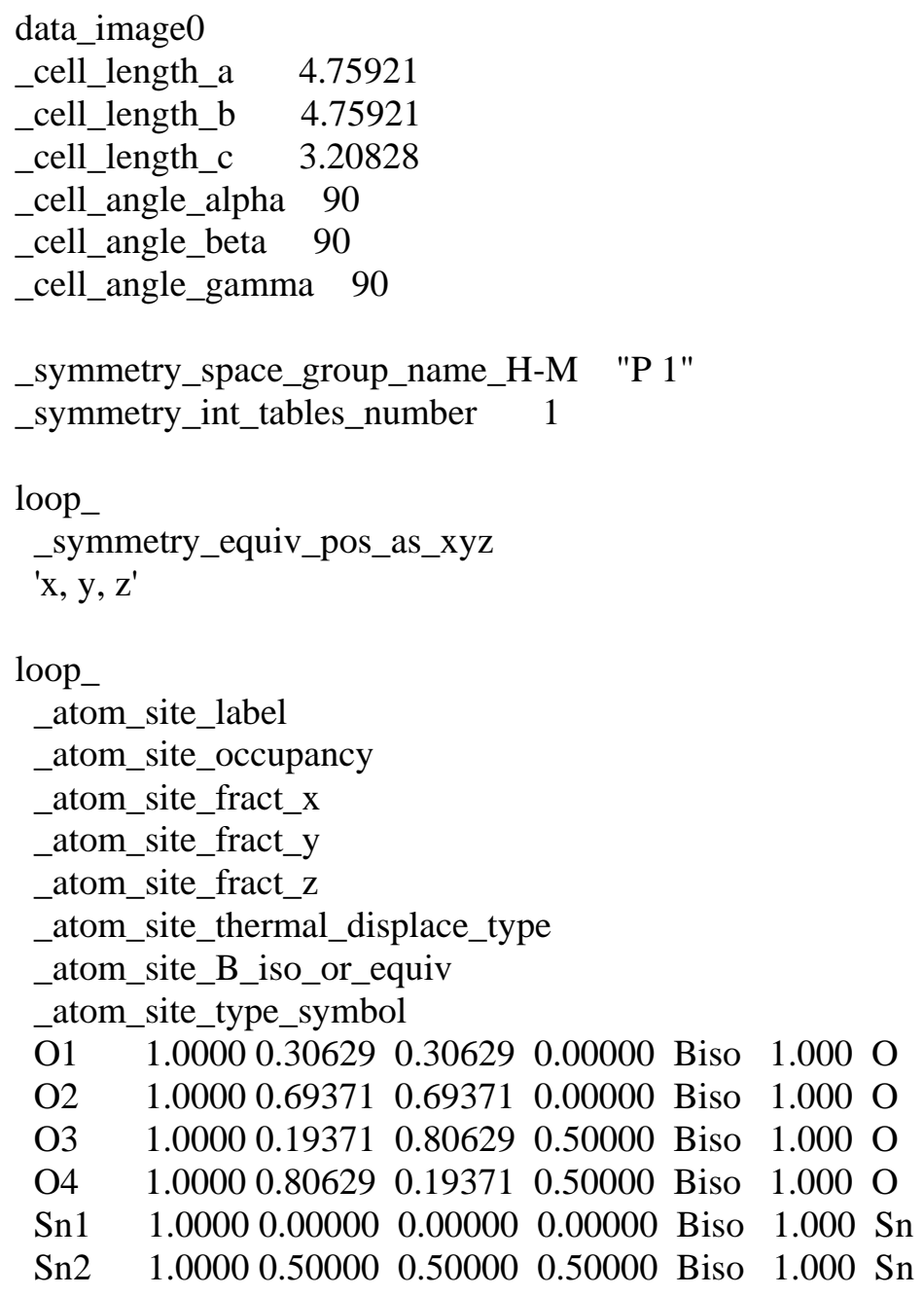

